\title{
Changes to virus taxonomy and to the International Code of Virus Classification and Nomenclature ratified by the International Committee on Taxonomy of Viruses (2021)
}

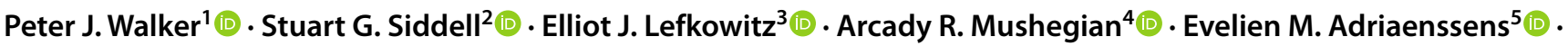

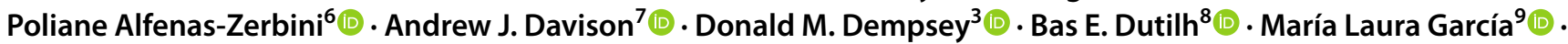

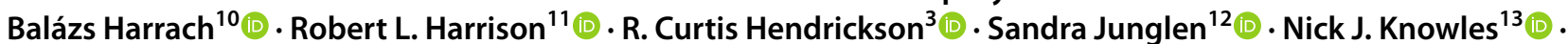 \\ Mart Krupovic $^{14}$ (D) . Jens H. Kuhn ${ }^{15}$ (1) - Amy J. Lambert ${ }^{16}$ (1) Małgorzata Łobocka ${ }^{17}$ (1) - Max L. Nibert ${ }^{18}$ (D)

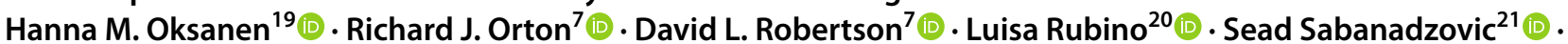 \\ Peter Simmonds ${ }^{22}$ (1) . Donald B. Smith ${ }^{22}$ (1) . Nobuhiro Suzuki ${ }^{23}$ (1) . Koenraad Van Dooerslaer ${ }^{24}(1)$. \\ Anne-Mieke Vandamme ${ }^{25,26}$ (1) Arvind Varsani ${ }^{27}$ (i) Francisco Murilo Zerbini ${ }^{28}\left({ }_{0}\right.$
}

Published online: 6 July 2021

(c) This is a U.S. government work and not under copyright protection in the U.S.; foreign copyright protection may apply 2021

\begin{abstract}
This article reports the changes to virus taxonomy approved and ratified by the International Committee on Taxonomy of Viruses (ICTV) in March 2021. The entire ICTV was invited to vote on 290 taxonomic proposals approved by the ICTV Executive Committee at its meeting in October 2020, as well as on the proposed revision of the International Code of Virus Classification and Nomenclature (ICVCN). All proposals and the revision were ratified by an absolute majority of the ICTV members. Of note, ICTV mandated a uniform rule for virus species naming, which will follow the binomial 'genus-species' format with or without Latinized species epithets. The Study Groups are requested to convert all previously established species names to the new format. ICTV has also abolished the notion of a type species, i.e., a species chosen to serve as a name-bearing type of a virus genus. The remit of ICTV has been clarified through an official definition of 'virus' and several other types of mobile genetic elements. The ICVCN and ICTV Statutes have been amended to reflect these changes.
\end{abstract}

\section{Introduction}

Changes to virus taxonomy (the universal scheme of virus classification of the International Committee on Taxonomy of Viruses [ICTV]) take place annually and are the result of a multi-stage process. In accordance with the ICTV Statutes (https://ictv.global/statutes.asp), taxonomic proposals (TaxoProps) submitted to the ICTV Executive Committee (EC) undergo a review process that involves input from the ICTV Study Groups (SGs) and Subcommittees (SCs), other interested virologists, and the EC. After final approval by the EC, proposals are presented for ratification to the full ICTV membership by posting on the ICTV website (https://ictv. global/), followed by an electronic vote.

Handling Editor: Tim Skern.

Arcady R. Mushegian

mushegian2@gmail.com

Extended author information available on the last page of the article
The latest set of proposals approved by the EC was made available on the ICTV website in February 2021 (see https:// ictv.global/proposal/MSL36/ for all proposals combined into a single zip file, and use the links provided in the References to access individual proposals). A list of proposals was then emailed on 3rd February 2021 to the 176 members of ICTV, namely the EC Members, Life Members, ICTV SC Members including the SG chairs, and ICTV National Representatives. Members were then requested to vote on whether to ratify the taxonomic proposals. Voting closed on 5th March 2021. 


\section{Changes to virus taxonomy, to the International Code of Virus Classification and Nomenclature, and to the ICTV Statutes ratified in 2021}

All proposals were ratified by ICTV members, in every case receiving an absolute majority of votes; at least $60 \%$ of eligible voters approved each proposal. A summary of the taxonomy changes enacted by ratification of the proposals is provided in Table 1. Each proposal is cited and listed in the References to acknowledge the authors' efforts and to provide links to the specific proposal on the ICTV website [1-297]. These documents remain available for those who wish to see the full details of the proposals. A ratified nontaxonomic proposal 2020.002G [3] specifies a novel procedure for expedited correction of unintended errors that may occur during proposal writing but clearly do not reflect the intention of the proposers, as described in the submitted proposal documentation. Examples of such errors include assignment of a lower-rank taxon to an incorrect higher rank, inadvertent introduction of duplicate taxon names, or assignment of a misspelled taxon name. Since the changes ratified by ICTV take effect immediately, this mechanism was used to correct seven proposals that relate to proposals ratified in March 2021 but were found to contain errors upon crosschecking [18, 53, 54, 66, 254, 255, 297].

A notable change approved this year is the adoption of a uniform binomial format for naming of virus species [1]. Previously, virologists have not been required to follow the convention of species naming that has been established in other fields of biology, whereby the species name consists of the genus name separated by a space from a species epithet that identifies the species within that genus. Over the last several decades, the case for a standardized nomenclature for virus species has been acknowledged by the ICTV and by many other virologists, but a uniform format of the species names had not been adopted (see references [298-304] for an incomplete but relatively representative account of the opinion spectrum). The case for a binomial nomenclature was presented by the ICTV Executive Committee in 2018 and 2020, and the advantages and disadvantages of different naming formats within the binomial framework were discussed [305, 306]. The EC then invited comments on this position from the virology community. Comments from ICTV SGs were also solicited and received by the EC SCs. Debates of the merits of various forms of binomial nomenclature took place in social media (e.g., 307) and in journal publications [308, 309]. Taking these discussions and comments into account, ICTV ratified the adoption of a standardized binomial system, which may follow either Latinized or free form formats [1]. The ICTV SGs are now charged with conversion of all existing virus species names to the new binomial form; the process has started already and is expected to be completed in time to be considered by an EC meeting planned for 2023. It is worth emphasizing that this system concerns only the names of virus species but does not affect the names of viruses.

Another general proposal [2] was the abolition of the requirement for a type species-a designated nomenclatural type for each genus. The ICTV noted that the requirement for type species in virus taxonomy was largely historical. Most importantly, the ICVCN defines virus species as follows: "A species is a monophyletic group of viruses whose properties can be distinguished from those of other species by multiple criteria", suggesting that the emphasis on "defining" a genus has been shifting from the properties of any particular member to the closeness of molecular and ecological relationships between all genus members. The ICTV
Table 1 Summary of taxonomic changes approved in March 2021

\begin{tabular}{llllllll}
\hline Rank & Total, MSL-35 & New & Moved & Abolished & Renamed & Merged & Total, MSL-36 $^{\mathrm{b}}$ \\
\hline Realm & 4 & 2 & 0 & 0 & 0 & 0 & 6 \\
Kingdom & 9 & 1 & 0 & 0 & 0 & 0 & 10 \\
Phylum & 16 & 1 & 0 & 0 & 0 & 0 & 17 \\
Class & 36 & 3 & 0 & 0 & 1 & 0 & 39 \\
Order & 55 & 4 & 1 & 0 & 1 & 0 & 59 \\
Family & 168 & 22 & 5 & 1 & 1 & 0 & 189 \\
Subfamily & 103 & $33^{\mathrm{c}}$ & 2 & 0 & 0 & 0 & 136 \\
Genus & 1,421 & 807 & 69 & $3^{\mathrm{c}, \mathrm{d}}$ & 10 & 1 & 2,224 \\
Subgenus & 68 & $2^{\mathrm{d}}$ & 0 & 0 & 2 & 0 & 70 \\
Species & 6,590 & 2,562 & 126 & 30 & 215 & 12 & 9,110 \\
\hline
\end{tabular}

\footnotetext{
${ }^{\text {a }}$ Total number of taxa in the ICTV Master Species List prior to 2021 ratification

${ }^{\mathrm{b}}$ Total number of taxa now recognized and reported in the ICTV Master Species List

${ }^{c}$ One new subfamily promoted from genus

${ }^{\mathrm{d}}$ One new subgenus demoted from genus
} 
concluded that the concept of a type species no longer serves a clear and useful purpose in virology and voted to abolish it.

One more proposal [4] has clarified the remit of the ICTV. In addition to viruses, ICTV has been classifying viroids and virus satellites, though none of these mobile genetic elements, including viruses themselves, have been operationally defined, leaving the limits of the ICTV scope unclear. This has been clarified now by giving operational definitions of viruses sensu stricto, viriforms, viroids, and satellites. These entities together constitute the virosphere and are within the remit of the ICTV responsibilities for their classification, taxonomy and nomenclature. The historical name of the organization - the International Committee on Taxonomy of Viruses-remains unchanged.

\section{Conclusion}

All proposals submitted to ratification were ratified by an absolute majority of the ICTV, and the changes are now part of official ICTV taxonomy. An up-to-date list of all approved taxa, which now includes more than 9000 virus species, can be found on the ICTV website: see https://ictv.global/propo sal/MSL36/ for the Master Species List and https://ictv. global/vmr/ for the Virus Metadata Resource.

Funding E.M.A. gratefully acknowledges funding by the U.K. Biotechnology and Biological Sciences Research Council (BBSRC); this research was funded by the BBSRC Institute Strategic Programme Gut Microbes and Health BB/R012490/1 and its constituent projects BBS/E/F/000PR10353 and BBS/E/F/000PR10356. A.J.D. is supported by the U.K. Medical Research Council (MC_UU_12014/3). B.E.D. is supported by Netherlands Organization for Scientific Research (NWO) Vidi grant 864.14.004. B.H. is supported by the National Research, Development and Innovation Office-NKFIH (NN128309). N.J.K. is partially supported by core funding provided by the Biotechnology and Biological Sciences Research Council, UK. Mention of trade names or commercial products in this publication is solely for the purpose of providing specific information and does not imply recommendation or endorsement by the U.S. Department of Agriculture. USDA is an Equal Opportunity Provider and Employer. This work was supported in part through Laulima Government Solutions, LLC prime contract with the US National Institute of Allergy and Infectious Diseases (NIAID) under Contract No. HHSN272201800013C. J.H.K. performed this work as an employee of Tunnell Government Services (TGS), a subcontractor of Laulima Government Solutions, LLC under Contract No. HHSN272201800013C. A.R.M. is a Program Director at the U.S. National Science Foundation (NSF); the statements and opinions expressed herein are made in a personal capacity and do not constitute endorsement by NSF or the government of the United States. H.M.O was supported by the University of Helsinki and Academy of Finland by funding for FINStruct and Instruct Centre FI, part of Biocenter Finland and Instruct-ERIC. D.L.R. is supported by the U.K. Medical Research Council (MC_UU_1201412). R.J.O. and D.B.S. are supported by the Wellcome Trust (WT108418AIA). S.S. acknowledges partial support from the Special Research Initiative (MAFES), Mississippi State University, and from the National Institute of Food and Agriculture, US Department of Agriculture, Hatch Project 1021494.
Except for Donald M. Dempsey, R. Curtis Hendrickson, Richard J. Orton and Donald B. Smith, the authors were members of the ICTV Executive Committee during the relevant period. The views and conclusions contained in this document are those of the authors and should not be interpreted as necessarily representing the official policies, either expressed or implied, of the US Department of Health and Human Services, or of the institutions and companies affiliated with the authors.

\section{Declarations}

Conflict of interest The authors declare no conflicts of interest

Human and animal rights statement None of the work reported involved research on human participants or animals. All authors have contributed to this work and agreed to its publication.

\section{References}

1. Adriaenssens EM, Dutilh BE, Harrach B, Junglen S, Kropinski AM, Krupovic M, Kuhn JH, Mushegian A, Postler TS, Rubino L, Sabanadzovic S, Simmonds P, Varsani A, Zerbini M (2018) Modify the International Code of Virus Classification and Nomenclature (ICVCN) to prospectively mandate a uniform genus-species type virus species naming format https://ictv. global/ictv/proposals/2018.001G.R.binomial_species.pdf. kuhnjens@mail.nih.gov

2. Siddell SG, Lefkowitz EJ, Walker PJ (2020) Abolish type species https://ictv.global/ictv/proposals/2020.001G.R.Abolish_type_ species.pdf. stuart.siddell@bristol.ac.uk

3. Walker PJ, Lefkowitz EJ, Mushegian A, Siddell SG, Davison AJ (2020) Adopt an expedited procedure for correction of unintended errors in ratified virus taxonomy https://ictv.global/ictv/ proposals/2020.002G.R.Expedited_error_correction.pdf. peter. walker@uq.edu.au

4. Kuhn JH, Dolja VV, Krupovic M, Adriaenssens EM, Di Serio F, Dutilh BE, Flores R, Harrach B. Mushegian A, Owens B, Randles J, Rubino L, Sabanadzovic S, Simmonds P, Varsani A, Zerbini M, Koonin EV (2020) Expand, amend, and emend the International Code of Virus Classification and Nomenclature (ICVCN; "the Code") and the Statutes to clearly define the remit of the ICTV https://ictv.global/ictv/proposals/2020.005G.R. Code_and_Statute_Change.pdf. kuhnjens@mail.nih.gov

5. Kuhn JH (2021) Correct mistakes inadvertently introduced into the ICVCN in $2020 \mathrm{https}: / /$ ictv.global/ictv/proposals/2020.006G. Code_Correction.docx. kuhnjens@mail.nih.gov

6. Chen YJ, Qi BX, Zheng GL, Zhang Y, Su R, Li CY (2020) Create one new species (Spodoptera exigua multiple nucleopolyhedrovirus $B$ ) and rename one species in the genus Alphabaculovirus (Lefavirales: Baculoviridae) https://ictv.global/ictv/proposals/ 2020.001D.R.Alphabaculovirus_1nsp.zip.cyli@qau.edu.cn

7. Kolb AW, Brandt CR (2020) Create two new species in the genus Simplexvirus (Herpesvirales: Herpesviridae) https://ictv.global/ ictv/proposals/2020.002D.R.Herpesviridae_2nsp.zip. awkolb@ wisc.edu

8. Tisza MJ, Starrett GJ, Buck CB (2020) Create one new class (Polintoviricetes), one new order (Orthopolintovirales), one new family (Adintoviridae), two genera and two species in the phylum Preplasmaviricota https://ictv.global/ictv/proposals/2020. 003D.R.Adintoviridae.zip.buckc@ mail.nih.gov

9. Doszpoly A, Kaján GL, Puentes R, Perretta A (2020) Create one new species in the genus Lymphocystivirus (Pimascovirales: 
Iridoviridae) https://ictv.global/ictv/proposals/2020.004D.R. Iridoviridae_1nsp.zip. andor.doszpoly@gmail.com

10. Harrison RL, Herniou EA, Jehle JA, Theilmann DA, Burand JP, Krell PJ, van Oers MM, Vlak JM (2020) Create two new genera and eight new species (Lefavirales: Nudiviridae) https://ictv. global/ictv/proposals/2020.005D.R.Nudiviridae_2ngen_8nsp. zip. Robert.L.Harrison@ars.usda.gov

11. Harrison RL, Herniou EA, Jehle JA, Theilmann DA, Burand JP, Krell PJ, van Oers MM (2020) Create one new class (Naldaviricetes) including one new order (Lefavirales) for four families of arthropod-specific large DNA viruses https://ictv.global/ictv/ proposals/2020.006D.R.Naldaviricetes.zip. Robert.L.Harrison@ ars.usda.gov

12. Gatherer D, Benkő M, Brandt CR, Bryant NA, das Neves CG, Dastjerdi A, Depledge DP, Doszpoly A, Gompels UA, Hartley CA, Inoue N, Jarosinski KW, Kaul R, Lacoste V, Norberg P, Origgi FC, Orton RJ, Pellett PE, Schmid DS, Spatz SJ, Stewart JP, Szpara ML, Trimpert J, Vaz P, Waltzek TB, Davison AJ (2020) Create four new genera (Quwivirus, Patagivirus, Bossavirus, Manticavirus) including five new species and two previously unassigned species, and one new species in the genus Varicellovirus (Herpesvirales: Herpesviridae) https://ictv.global/ictv/ proposals/2020.007D.R.Herpesviridae_4ngen_6nsp.zip. andrew. davison@glasgow.ac.uk

13. Tarján ZL, Pénzes JJ, Doszpoly A, Harrach B, Benkő M (2020) Create one new genus (Testadenovirus) and six new species (Rowavirales: Adenoviridae) https://ictv.global/ictv/proposals/ 2020.008D.R.Adenoviridae_1ngen_6nsp.zip.maribenko@gmail. com

14. Fisher M, Harrison T, Nebroski M, Kruczkiewicz P, Rothenburger J, Ambagala A, Macbeth B, Lung O (2020) Create one new species in the genus Circovirus (Circlivirales: Circoviridae) https://ictv.global/ictv/proposals/2020.009D.R.Circovirus_1nsp. zip. oliver.lung@ canada.ca

15. Varsani A, Rosario K, Delwart E, Segales J, Breitbart M (2020) Create six new species in the genus Circovirus and one new species in the genus Cyclovirus (Circlivirales: Circoviridae) https:// ictv.global/ictv/proposals/2020.010D.R.Circoviridae_7nsp.zip. Arvind.varsani@asu.edu

16. Penzes JJ, Canuti M, Söderlund-Venermo M, Eis-Huebinger AM, Ogliastro M, Harrach B (2020) Create three new genera and 19 new species (Piccovirales: Parvoviridae) https://ictv.global/ictv/ proposals/2020.011D.R.Parvoviridae_3ngen_20nsp.zip. judit. penzes@ufl.edu

17. Hepojoki J, Hetzel U, Paraskevopoulou S, Drosten C, Harrach B, Zerbini M, Koonin EV, Krupovic M, Dolja V, Kuhn JH (2020) Create one new realm (Ribozyviria) including one new family (Kolmioviridae) including genus Deltavirus and seven new genera for a total of 15 species https://ictv.global/ictv/proposals/ 2020.012D.R.Ribozyviria.zip. jussi.hepojoki@helsinki.fi

18. Harrach B (2020) Correct the misspelt name of one species (Bluegill hepatitis B virus) in the genus Metahepadnavirus (Blubervirales: Hepadnaviridae) https://ictv.global/ictv/proposals/ 2020.013D.R.Metahepadnavirus_1sp.zip.balazs.harrach@gmail. com

19. Kraberger S, Opriessnig T, Varsani A (2020) Create nine new species in the genus Gyrovirus (Anelloviridae) https://ictv.global/ ictv/proposals/2020.014D.R.Gyrovirus_9nsp.zip. Arvind.varsani@asu.edu

20. Varsani A, Kraberger S (2020) Create 17 genera and new 80 species (Anelloviridae) https://ictv.global/ictv/proposals/2020. 015D.R.Anelloviridae_17ngen_80nsp.zip.Arvind.Varsani@asu. edu

21. Varsani A, Krupovic M (2020) Create six new genera and 42 new species (Cremevirales: Smacoviridae) https://ictv.global/
ictv/proposals/2020.016D.R.Smacoviridae_6ngen_42nsp.zip. Arvind.varsani@asu.edu

22. Chinchar VG, Williams T, Jancovich J, Hick P, Zhang QY, Marschang R, Whittington R, Waltzek T, Subramaniam K, Ince IA, Jie H (2020) Create one new species in the genus Ranavirus (Pimascovirales: Iridoviridae) https://ictv.global/ictv/proposals/ 2020.017D.R.Ranavirus_1nsp.zip.vchinchar@umc.edu

23. Chinchar VG, Waltzek T, Subramaniam K, Faria VG, Ebert D, Jancovich J, Hick P, Zhang QY, Marschang R, Whittington R, Williams T, Ince IA, Jie H (2020) Create one new genus (Daphniairidovirus) including one new species in subfamily Betairidovirinae (Primascovirales: Iridoviridae) https://ictv.global/

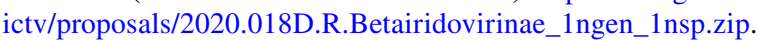
vchinchar@umc.edu

24. Calvignac-Spencer S, Carr M, Daugherty MD, Feltkamp MCW, Lauber C, Moens U, Verschoor EJ, Ehlers B (2020) Create two new genera and 15 new species (Sepolyvirales: Polyomaviridae) https://ictv.global/ictv/proposals/2020.019D.R.Polyomaviridae_ 2ngen_15nsp.zip.ehlersb@rki.de

25. Walker PJ, Blasdell KR, Breyda R, Dietzgen RG, Fooks AR, Freitas-Astúa J, Kondo H, Kuzmin IV, Tesh RB, Vasilakis N, Whitfield AE (2020) Create one new genus (Alpharicinrhavirus) including three new species (Mononegavirales: Rhabdoviridae) https://ictv.global/ictv/proposals/2020.001M.R.Alpharicinrhavi rus.zip.peter.walker@uq.edu.au

26. Bojko J, Smagghe G, Kuhn JH (2020) Create two new species in the genus Lincruvirus (Bunyavirales: Cruliviridae) https:// ictv.global/ictv/proposals/2020.002M.R.Lincruvirus_2nsp.zip. Jamie.Bojko@ufl.edu

27. Walker PJ, Blasdell KR, Breyta R, Dietzgen RG, Freitas-Astúa J, Kuzmin IV, Tesh RB, Tordo N, Vasilakis N, Shi M (2020) Create ten new species in the genus Sigmavirus (Mononegavirales: Rhabdoviridae) https://ictv.global/ictv/proposals/2020.003M.R. Sigmavirus_10nsp.zip.peter.walker@uq.edu.au

28. Koloniuk I, Fránová J (2020) Create three new species in the genus Cytorhabdovirus (Mononegavirales: Rhabdoviridae) https://ictv.global/ictv/proposals/2020.004M.R.Cytorhabdo virus_3nsp.zip.koloniuk@umbr.cas.cz

29. Walker PJ, Blasdell KR (2020) Create three new species in the genus Ephemerovirus (Mononegavirales: Rhabdoviridae) https:// ictv.global/ictv/proposals/2020.005M.R.Ephemerovirus_3nsp. zip.peter.walker@uq.edu.au

30. Kuhn JH (2020) Correction of errors inadvertently introduced into phylum Negarnaviricota taxonomy in 2019 https://ictv. global/ictv/proposals/2020.006M.R.Corrections.zip.kuhnjens@ mail.nih.gov

31. Walker PJ, Blasdell KR, Dietzgen RG, Freitas-Astúa J, Kondo H, Kuzmin IV, Tesh RB, Vasilakis N, Whitfield AE (2020) Create three new species in the genus Ohlsrhavirus (Mononegavirales: Rhabdoviridae) https://ictv.global/ictv/proposals/2020.007M.R. Ohlsrhavirus_3nsp.zip.peter.walker@uq.edu.au

32. Walker PJ, Blasdell KR, Dietzgen RG, Fooks AR, Freitas-Astúa J, Kondo H, Kuzmin IV, Tesh RB, Vasilakis N, Whitfield AE (2020) Create one new species (Dillard sunrhavirus) in the genus Sunrhavirus (Mononegavirales: Rhabdoviridae) https:// ictv.global/ictv/proposals/2020.008M.R.Sunrhavirus_1nsp.zip. peter.walker@uq.edu.au

33. Walker PJ, Tesh RB, Vasilakis N, Tu, C (2020) Create one new species and rename one species in the genus Vesiculovirus (Mononegavirales: Rhabdoviridae) https://ictv.global/ictv/proposals/ 2020.009M.R.Vesiculovirus_1nsp_ren1sp.zip.peter.walker@ uq.edu.au

34. Walker PJ, Blasdell KR, Dietzgen RG, Kondo H, Kuzmin IV, Tesh RB, Vasilakis N, Whitfield AE (2020) Create one new species (Hyalomma lostrhavirus) in the genus Lostrhavirus 
(Mononegavirales: Rhabdoviridae) https://ictv.global/ictv/ proposals/2020.010M.R.Lostrhavirus_1nsp.zip.peter.walker@ uq.edu.au

35. Balkema-Buschmann A, Dundon WG, Duprex WP, Easton AJ, Fouchier RAM, Kurath G, Lamb RA, Lee B, Rima BK, Rota PA, Wang LF (2020) Create one new species (Avian metaavulavirus 22) in the genus Metaavulavirus (Mononegavirales: Paramyxoviridae) https://ictv.global/ictv/proposals/2020. 011M.R.Metaavulavirus_1nsp.zip.b.rima@qub.ac.uk

36. Hughes HR (2020) Create 16 new species and abolish one species in the genus Orthobunyavirus (Bunyavirales: Peribunyaviridae) https://ictv.global/ictv/proposals/2020.012M.R.Ortho bunyavirus_16new_sp_abolish_1sp.zip.LTR8@cdc.gov

37. Dietzgen RG, Freitas-Astúa J, Kondo H, Whitfield AE, Bejerman N, Debat H, Walker PJ (2020) Create two new species in the genus Alphanucleorhabdovirus, three new species in the genus Betanucleorhabdovirus, two new species in the genus Cytorhabdovirus and two new species in the genus Varicosavirus (Mononegavirales: Rhabdoviridae) https://ictv.global/ ictv/proposals/2020.013M.R.Plant_rhabdoviruses_9nsp.zip. r.dietzgen@uq.edu.au

38. Walker PJ, Blasdell KR, Dietzgen RG, AR, Fooks, FreitasAstúa J, Kondo H, Kuzmin IV, Tesh RB, Vasilakis N, Whitfield AE (2020) Create one new genus (Alphapaprhavirus) including two new species (Mononegavirales: Rhabdoviridae) https:// ictv.global/ictv/proposals/2020.014M.R.Alphapaprhavirus_ 1gen2sp.zip.peter.walker@uq.edu.au

39. Walker PJ, Blasdell KR, Dietzgen RG, Fooks AR, FreitasAstúa J, Kondo H, Kuzmin IV, Tesh RB, Vasilakis N, Whitfield AE (2020) Create one new genus (Merhavirus) including two new species (Mononegavirales: Rhabdoviridae) https://ictv. global/ictv/proposals/2020.015M.R.Merhavirus_1gen2nsp. zip.peter.walker@uq.edu.au

40. Walker PJ, Blasdell KR, Breyta R, Dietzgen RG, Fooks AR, Freitas-Astúa J, Kondo H, Kurath G, Kuzmin IV, Stone DM, Tesh RB, Tordo N, Vasilakis N, Whitfield AE (2020) Create three new subfamilies in the family Rhabdoviridae (Mononegavirales) https://ictv.global/ictv/proposals/2020.016M.R.Rhabd oviridae_3subfam.zip.peter.walker@uq.edu.au

41. Shi Z-L, Kuhn JH, Lukashevich IS, Clegg JCS, Gonzalez J-PJ, de la Torre JCC, Hepojoki J, Salvato M, Stenglein M, Buchmeier MJ, Charrel RN, Radoshitzky SRR, Günther S, Romanowski V, de Lamballerie X, Sironi M, Agwanda B (2020) Create one new species in genus Mammarenavirus (Bunyavirales: Arenaviridae) https://ictv.global/ictv/propo sals/2020.017M.R.Mammarenavirus.zip.zlshi@wh.iov.cn

42. Mordecai GJ, Miller KM, Di Cicco E, Schulze AD, Kaukinen KH, Ming TJ, Li S, Tabata A, Teffer A, Patterson DA, Ferguson HW, Suttle CA (2020) Create one new species in genus Antennavirus (Bunyavirales: Arenaviridae) https://ictv.global/ ictv/proposals/2020.018M.R.Antennavirus.zip. gidmord@ gmail.com

43. Hepojoki J (2020) Create two new species in the genus Hartmanivirus (Bunyavirales: Arenaviridae) https://ictv.global/ictv/ proposals/2020.019M.R.Hartmanivirus.zip.jussi.hepojoki@ helsinki.fi

44. Ballinger MJ, Hall RA, Langevin SA, Pauvolid-Corrêa A, Paraskevopoulou S, Drosten C, Junglen S (2020) Create four new species in the genus Orthophasmavirus, create two new species in the genus Feravirus and create one new genus (Hymovirus) including two new species (Bunyavirales: Phasmaviridae) https://ictv.global/ictv/proposals/2020.020M.R.Phasmaviridae. zip. sandra.junglen@ charite.de

45. Walker PJ, Bennett AJ, Kuhn JH, Goldberg TL (2020) Create one new species (Bughendera ledantevirus) in the genus Ledantevirus (Mononegavirales: Rhabdoviridae) https://ictv.global/ictv/
proposals/2020.021M.R.Ledantevirus_1nsp.zip.peter.walker@ uq.edu.au

46. Marklewitz M, Tchouassi DP, Torto B, Sang R, Junglen S (2020) Create four new species in the genus Phlebovirus (Bunyavirales: Phenuiviridae) https://ictv.global/ictv/proposals/2020.022M.R. Phlebovirus_4sp.zip.sandra.junglen@ charite.de

47. Walker PJ, Blasdell KR, Dietzgen RG, Freitas-Astúa J, Kondo H, Kurath G, Kuzmin IV, Tesh RB, Tordo N, Vasilakis N, Whitfield AE (2020) Create seven new genera (Alphacrustrhavirus, Alphadrosrhavirus, Alphahymrhavirus, Betahymrhavirus, Betanemrhavirus, Betapaprhavirus and Betaricinrhavirus) including 16 new species (Mononegavirales: Rhabdoviridae) https://ictv.global/ictv/proposals/2020.023M.R.Rhabdoviridae_ 7ngen_16nsp.zip.peter.walker@uq.edu.au

48. Dietzgen RG, Kondo H, Kuhn JH, Vasilakis N, Jiang D, Junglen S (2020) Create one new species in the genus Nyavirus and four new species in one new genus Formivirus (Mononegavirales: Nyamiviridae) https://ictv.global/ictv/proposals/2020.024M.R. Nyamiviridae_1ngen_5nsp.zip.r.dietzgen@uq.edu.au

49. Maes P, Bradfute P, Calisher CH, Klempa B, Klingström J, Laenen L, Song JW, Zhang YZ (2020) Create five new species in subfamily Mammantavirinae (Bunyavirales: Hantaviridae) https://ictv.global/ictv/proposals/2020.025M.R.Hantaviridae_ 5nsp.zip.piet.maes@kuleuven.be

50. Di Paola N, Dheilly NM, Kuhn JH, Junglen S, Paraskevopoulou S, Postler TS, Shi M (2020) Reorganize the order to include four new families, 18 new genera, and 22 new species (Jingchuvirales) https://ictv.global/ictv/proposals/2020.026M.R.Jingchuvir ales.zip.nicholas.dipaola.ctr@mail.mil

51. Marklewitz M, Paraskevopoulou S, Alkhovsky SV, Avšič-Županc T, Bente D, Bergeron É, Burt F, Ergünay K, Garrison AR, Hewson R, Mirazami A, Palacios G, Papa A, Pawęska JT, Sall AA, Spengler J, Di Paola N, Kuhn JH (2020) Create four new genera and 30 new species (Bunyavirales: Nairoviridae) https://ictv. global/ictv/proposals/2020.027M.R.Nairoviridae_4ngen_30nsp. zip.marco.marklewitz@charite.de

52. Marklewitz M, Paraskevopoulou S, Briese T, Charrel RN, Choi I-R, De Lamballerie X, Ebihara H, Gāo GF, Groschup MH, Jonson GB, Nunes MRT, Palacios G, Sasaya T, Shirako Y, Song J-W, Wèi T, Zerbini M, Zhou X, Kuhn JH (2020) Create one new genus and 16 new species (Bunyavirales: Phenuiviridae) https://ictv.global/ictv/proposals/2020.029M.R.Phenuiviridae_ 1gen16sp.zip.marco.marklewitz@ charite.de

53. Kuhn JH (2021) Correct errors inadvertently introduced into phylum Negarnaviricota taxonomy https://ictv.global/ictv/proposals/ 2020.030M.R.Negarnaviricota_corrections.zip.kuhnjens@mail. nih.gov

54. Walker PJ (2021) Correct an error in the spreadsheet for ratified proposal 2020.016M (Mononegavirales: Rhabdoviridae) https:// ictv.global/ictv/proposals/2020.031M.R.Corrections_Rhabdoviri dae.zip.peter.walker@uq.edu.au

55. Zell R, Gorbalenya AE, Hovi T, Knowles NJ, Lindberg AM, Oberste MS, Palmenberg AC, Reuter G, Simmonds P, Skern T, Tapparel C, Wolthers KC, Woo PCY (2020) Create one new genus (Caecilivirus) including one new species (Picornavirales: Picornaviridae) https://ictv.global/ictv/proposals/2020.001S.R. Caecilivirus_1ngen1nsp.zip.roland.zell@med.uni-jena.de

56. Zell R, Gorbalenya AE, Hovi T, Knowles NJ, Lindberg AM, Oberste MS, Palmenberg AC, Reuter G, Simmonds P, Skern T, Tapparel C, Wolthers KC, Woo PCY (2020) Create three new genera (Danipivirus, Pygoscepivirus, Rajidapivirus) including three new species (Picornavirales: Picornaviridae) https://ictv. global/ictv/proposals/2020.002S.R.Danipi-Pygoscepi-Rajidapivi rus_3ngen3nsp.zip.roland.zell@med.uni-jena.de

57. Zell R, Gorbalenya AE, Hovi T, Knowles NJ, Lindberg AM, Oberste MS, Palmenberg AC, Reuter G, Simmonds P, Skern T, 
Tapparel C, Wolthers KC, Woo PCY (2020) Create one new species (Fipivirus F) in the genus Fipivirus (Picornavirales: Picornaviridae) https://ictv.global/ictv/proposals/2020.003S.R. Fipivirus_1nsp.zip.roland.zell@med.uni-jena.de

58. Zell R, Gorbalenya AE, Hovi T, Knowles NJ, Lindberg AM, Oberste MS, Palmenberg AC, Reuter G, Simmonds P, Skern T, Tapparel C, Wolthers KC, Woo PCY (2020) Create one new species (Grusopivirus $C$ ) in the genus Grusopivirus (Picornavirales: Picornaviridae) https://ictv.global/ictv/proposals/2020.004S.R. Grusopivirus_1nsp.zip.roland.zell@med.uni-jena.de

59. Zell R, Gorbalenya AE, Hovi T, Knowles NJ, Lindberg AM, Oberste MS, Palmenberg AC, Reuter G, Simmonds P, Skern T, Tapparel C, Wolthers KC, Woo PCY (2020) Create one new species (Limnipivirus D) in the genus Limnipivirus (Picornavirales: Picornaviridae) https://ictv.global/ictv/proposals/2020.005S.R. Limnipivirus_1nsp.zip.roland.zell@med.uni-jena.de

60. Zell R, Gorbalenya AE, Hovi T, Knowles NJ, Lindberg AM, Oberste MS, Palmenberg AC, Reuter G, Simmonds P, Skern T, Tapparel C, Wolthers KC, Woo PCY (2020) Create one new genus (Marsupivirus) including one new species (Picornavirales: Picornaviridae) https://ictv.global/ictv/proposals/2020.006S.R. Marsupivirus_1ngen1nsp.zip.roland.zell@med.uni-jena.de

61. Zell R, Gorbalenya AE, Hovi T, Knowles NJ, Lindberg AM, Oberste MS, Palmenberg AC, Reuter G, Simmonds P, Skern T, Tapparel C, Wolthers KC, Woo PCY (2020) Create one new species (Mischivirus E) in the genus Mischivirus (Picornavirales: Picornaviridae) https://ictv.global/ictv/proposals/2020.007S.R. Mischivirus_1nsp.zip.roland.zell@med.uni-jena.de

62. Zell R, Gorbalenya AE, Hovi T, Knowles NJ, Lindberg AM, Oberste MS, Palmenberg AC, Reuter G, Simmonds P, Skern T, Tapparel C, Wolthers KC, Woo PCY (2020) Create one new species (Pemapivirus B) in the genus Pemapivirus (Picornavirales: Picornaviridae) https://ictv.global/ictv/proposals/2020.008S.R. Pemapivirus_1nsp.zip.roland.zell@med.uni-jena.de

63. Zell R, Gorbalenya AE, Hovi T, Knowles NJ, Lindberg AM, Oberste MS, Palmenberg AC, Reuter G, Simmonds P, Skern T, Tapparel C, Wolthers KC, Woo PCY (2020) Create one new species (Tropivirus B) in the genus Tropivirus (Picornavirales: Picornaviridae) https://ictv.global/ictv/proposals/2020.009S.R. Tropivirus_1nsp.zip.roland.zell@med.uni-jena.de

64. Tschá MK, Suzukawa AA, Gräf T, Piancini LDS, Silva AM, Faoro H, Riediger IN, Medeiros LC, Wowk PF, Zanluca C, Duarte dos Santos CN (2020) Create one new species (Caaingua virus) in the genus Alphavirus (Martellivirales: Togaviridae) https://ictv.global/ictv/proposals/2020.010S.R.Caaingua_virus_ 1nsp.zip. claudia.dossantos@ fiocruz.br

65. Bennett AJ, Paskey AC, Ebinger A, Kuhn JH, Bishop-Lilly KA, Beer M, Goldberg TL (2020) Create two new species and rename one species in genus Rubivirus (Hepelivirales: Matonaviridae) https://ictv.global/ictv/proposals/2020.011S.R.Rubivirus_nspp. zip. tony.goldberg@wisc.edu

66. Brinton MA (2020) Correct errors in previous proposals (Nidovirales: Arteriviridae) https://ictv.global/ictv/proposals/2020. 014S.R.Arteriviridae_corr.zip.mbrinton@gsu.edu

67. Dyall-Smith ML, Witte A, Oesterhelt D, Pfeiffer F (2020) Create two new species in the genus Myohalovirus (Caudovirales: Myoviridae) https://ictv.global/ictv/proposals/2020.001B.R.Myoha lovirus.zip.mike.dyallsmith@gmail.com

68. Dyall-Smith ML, Tang S-L (2020) Create one new genus (Haloferacalesvirus) including five new species (Caudovirales: Myoviridae) https://ictv.global/ictv/proposals/2020.002B.R. Haloferacalesvirus.zip.mike.dyallsmith@gmail.com

69. Lehman SM, Adriaenssens EM (2020) Abolish the species Pseudomonas virus 42 (Caudovirales: Myoviridae) https://ictv.global/ ictv/proposals/2020.003B.R.Abolish_Ps_virus_42.zip. susan. lehman@fda.hhs.gov
70. Adriaenssens EM, Tolstoy I, Moraru C, Kropinski AM, Turner D (2020) Abolish the genus Viunavirus, move six existing species to the genus Kuttervirus and create 30 new species (Caudovirales: Ackermannviridae) https://ictv.global/ictv/proposals/2020. 004B.R.Ackermannviridae_reassessment.zip.Phage.Canada@ gmail.com

71. Maina AN, Kropinski AM, Tolstoy I, Adriaenssens EM, Moraru C, Turner D (2020) Create six new genera (Caudovirales: Ackermannviridae) https://ictv.global/ictv/proposals/2020.005B.R. Ackermannviridae.zip.Phage.Canada@gmail.com

72. Adriaenssens EM, Tolstoy I, Kropinski AM, Moraru C (2020) Correct minor errors in the taxonomy of four actinobacteriophage-related genera (Caudovirales: Siphoviridae) https://ictv. global/ictv/proposals/2020.006B.R.Actinobacteriophages_corre ct_taxa.zip.Phage.Canada@gmail.com

73. Adriaenssens EM, Tolstoy I, Moineau S, Kropinski AM (2020) Create one new genus (Agmunavirus) including one species (Caudovirales: Siphoviridae) https://ictv.global/ictv/proposals/ 2020.007B.R.Agmunavirus.zip. Phage.Canada@gmail.com

74. Adriaenssens EM, Tolstoy I, Turner D, Kropinski AM (2020) Create one new genus (Alachuavirus) including one species (Caudovirales: Siphoviridae) https://ictv.global/ictv/proposals/ 2020.008B.R.Alachuavirus.zip.Phage.Canada@gmail.com

75. Krupovic M (2020) Create two new species in the genus Bottigliavirus (Ampullaviridae) https://ictv.global/ictv/proposals/2020. 009B.R.Ampullaviridae.zip. mart.krupovic@ pasteur.fr

76. Adriaenssens EM, Tolstoy I, Łobocka M, Moraru C, Barylski J, Tong Y, Kropinski AM, Turner D (2020) Create one new genus (Anjalivirus) including two species (Caudovirales: Podoviridae) https://ictv.global/ictv/proposals/2020.010B.R.Anjalivirus.zip. Phage.Canada@gmail.com

77. Adriaenssens EM, Tolstoy I, Turner D, Kropinski AM (2020) Create one new genus (Annadreamyvirus) including two species (Caudovirales: Siphoviridae) https://ictv.global/ictv/proposals/ 2020.011B.R.Annadreamyvirus.zip.Phage.Canada@gmail.com

78. Adriaenssens EM, Turner D, Tolstoy I, Kropinski AM (2020) Create one new genus (Arawnvirus) including one species (Caudovirales: Siphoviridae) https://ictv.global/ictv/proposals/2020. 012B.R.Arawnvirus.zip.Phage.Canada@gmail.com

79. Lehman SM, Petit MA, Lossouarn J, Tong Y, Tolstoy I, Adriaenssens EM (2020) Create ten new genera (Caudovirales: Siphoviridae) https://ictv.global/ictv/proposals/2020.013B.R. Assorted_Siphoviridae.zip.susan.lehman@fda.hhs.gov

80. Adriaenssens EM, Tolstoy I, Łobocka M, Moraru C, Barylski J, Tong Y, Kropinski AM (2020) Create one new genus (Astrithrvirus) including one species (Caudovirales: Podoviridae) https:// ictv.global/ictv/proposals/2020.014B.R.Astrithrvirus.zip. Phage. Canada@gmail.com

81. Adriaenssens EM, Tolstoy I, Turner D, Lueder M, Mahony J, Neve H, Moineau S, Kropinski AM (2020) Create one new genus (Audreyjarvisvirus) including seven species (Caudovirales: Siphoviridae) https://ictv.global/ictv/proposals/2020.015B.R. Audreyjarvisvirus.zip. Phage.Canada@gmail.com

82. Kauffman KM, Hussain FA, Yang J, Arevalo P, Brown JM, Chang WK, Van Insberghe D, Elsherbini J, Sharma RS, Cutler MB, Kelly L, Polz MF (2020) Create one new family (Autolykiviridae) of non-tailed dsDNA bacterial viruses in the double jelly roll fold major capsid lineage https://ictv.global/ictv/proposals/ 2020.016B.R.Autolykiviridae.zip. kmkauffm@buffalo.edu

83. Adriaenssens EM, Tolstoy I, Moraru C, Kropinski AM (2020) Create one new genus (Ayohtrevirus) including one species (Caudovirales: Myoviridae) https://ictv.global/ictv/proposals/2020. 017B.R.Ayohtrevirus.zip.Phage.Canada@gmail.com

84. Adriaenssens EM, Tolstoy I, Kropinski AM, Moraru C, Łobocka M (2020) Create one new subfamily (Azeredovirinae) including one new genus (Dubowvirus) (Caudovirales: Siphoviridae) 
https://ictv.global/ictv/proposals/2020.018B.R.Azeredovirinae. zip. lobocka@ibb.waw.pl

85. Adriaenssens EM, Tolstoy I, Łobocka M, Moraru C, Barylski J, Tong Y, Kropinski AM (2020) Create one new genus (Badaztecvirus) including two species (Caudovirales: Podoviridae) https://ictv.global/ictv/proposals/2020.019B.R.Badaztecvirus. zip.Phage.Canada@gmail.com

86. Adriaenssens EM, Turner D, Tolstoy I, Kropinski AM (2020) Split the genus Barnyardvirus into three genera, two of which (Konstantinevirus, Predatorvirus) are new (Caudovirales: Siphoviridae) https://ictv.global/ictv/proposals/2020.020B.R.Barny ardvirus.zip.Phage.Canada@gmail.com

87. Kropinski AM, Tolstoy I, Adriaenssens EM, Turner D, Barylski J (2020) Create one new genus (Beceayunavirus) including one new species (Caudovirales: Siphoviridae) https://ictv.global/ictv/ proposals/2020.021B.R.Beceayunavirus.zip. Jakub.Barylski@ gmail.com

88. Adriaenssens EM, Tolstoy I, Kropinski AM Barylski J (2020) Create one new genus (Becedseptimavirus) including one new species (Caudovirales: Myoviridae) https://ictv.global/ictv/propo sals/2020.022B.R.Becedseptimavirus.zip. Jakub.Barylski@ gmail.com

89. Adriaenssens EM, Tolstoy I, Turner D, Kropinski AM (2020) Create one new subfamily (Beephvirinae) including three new genera (Caudovirales: Podoviridae) https://ictv.global/ictv/propo sals/2020.023B.R.Beephvirinae.zip.Phage.Canada@gmail.com

90. Kropinski AM, Tolstoy I, Turner D, Adriaenssens EM (2020) Create one new genus (Borockvirus) including one new species (Caudovirales: Myoviridae) https://ictv.global/ictv/proposals/ 2020.024B.R.Borockvirus.zip.Phage.Canada@gmail.com

91. Adriaenssens EM, Tolstoy I, Kropinski AM, Moraru C, Łobocka M (2020) Create one new subfamily (Bronfenbrennervirinae) including the new genus Peeveelvirus (Caudovirales: Siphoviridae) https://ictv.global/ictv/proposals/2020.025B.R.Bronfenbre nnervirinae.zip. lobocka@ibb.waw.pl

92. Adriaenssens EM, Tolstoy I, Turner D, Kropinski AM (2020) Create one new genus (Burrovirus) including three new species (Caudovirales: Podoviridae) https://ictv.global/ictv/proposals/ 2020.026B.R.Burrovirus.zip.Phage.Canada@gmail.com

93. Adriaenssens EM, Tolstoy I, Kropinski AM, Barylski J (2020) Create one new genus (Camtrevirus) including three new species (Caudovirales: Siphoviridae) https://ictv.global/ictv/proposals/ 2020.027B.R.Camtrevirus.zip. Jakub.Barylski@gmail.com

94. Adriaenssens EM, Tolstoy I, Kropinski AM, Wittmann J, Buttimer C, Coffey A (2020) Create one new genus (Cbunavirus) including four new species (Caudovirales: Schitoviridae) https:// ictv.global/ictv/proposals/2020.028B.R.Cbunavirus.zip. jow12@ dsmz.de

95. Adriaenssens EM, Tolstoy I, Moraru C, Turner D, Lueder M, Mahony J, Neve H, Moineau S, Kropinski AM (2020) Create 34 new species in the genus Ceduovirus (Caudovirales: Siphoviridae) https://ictv.global/ictv/proposals/2020.029B.R.Ceduovirus. zip.Phage.Canada@gmail.com

96. Anany H, Moraru C, Turner D, Adriaenssens EM, Kropinski AM (2020) Create two new subfamilies and three new genera (Caudovirales: Chaseviridae) https://ictv.global/ictv/proposals/2020. 030B.R.Chaseviridae_new_taxa.zip.Phage.Canada@gmail.com

97. Adriaenssens EM, Tolstoy I, Turner, D Kropinski AM, Moraru C (2020) Reassessment of the genus Cheoctovirus and formation of one new genus Avanivirus (Caudovirales: Siphoviridae) https:// ictv.global/ictv/proposals/2020.031B.R.Cheoctovirus.zip. Phage. Canada@gmail.com

98. Adriaenssens EM, Tolstoy I, Turner D, Lueder M, Moineau S, Kropinski AM (2020) Create one new genus (Chertseyvirus) including one new species (Caudovirales: Siphoviridae) https://
ictv.global/ictv/proposals/2020.032B.R.Chertseyvirus.zip. Phage.Canada@gmail.com

99. Adriaenssens EM, Tolstoy I, Kropinski AM, Moraru C (2020) Create nine new species in the genus Chivirus (Caudovirales: Siphoviridae) https://ictv.global/ictv/proposals/2020.033B.R. Chivirus.zip.Phage.Canada@gmail.com

100. Kropinski AM, Tolstoy I, Adriaenssens EM, Moineau S, Mahony J, Lueder M, Neve H (2020) Create one new genus (Chopinvirus) including one new species (Caudovirales: Podoviridae) https:// ictv.global/ictv/proposals/2020.034B.R.Chopinvirus.zip. Phage. Canada@gmail.com

101. Adriaenssens EM, Tolstoy I, Turner D, Kropinski AM (2020) Taxonomic analysis of the myoviruses infecting Clostridioides difficile (Caudovirales: Myoviridae) https://ictv.global/ictv/ proposals/2020.035B.R.Clostridioides_myoviruses.zip. Phage. Canada@gmail.com

102. Adriaenssens EM, Tolstoy I, Turner D, Kropinski AM (2020) Create one new genus of Mycobacterium siphoviruses (Cornievirus) including one new species (Caudovirales: Siphoviridae) https://ictv.global/ictv/proposals/2020.036B.R.Cornievirus.zip. Phage.Canada@gmail.com

103. Adriaenssens EM, Tolstoy I, Turner D, Kropinski AM (2020) Correction of errors in the master species list (Caudovirales) https://ictv.global/ictv/proposals/2020.037B.R.Correction_MSL_ Errors.zip. Phage.Canada@gmail.com

104. Adriaenssens EM, Tolstoy I, Kropinski AM, Łobocka M (2020) Create one new genus (Coventryvirus) including seven new species (Caudovirales: Siphoviridae) https://ictv.global/ictv/propo sals/2020.038B.R.Coventryvirus.zip. lobocka@ibb.waw.pl

105. Adriaenssens EM, Tolstoy I, Turner D, Kropinski AM (2020) Create three new genera (Cukevirus, Indlulamithivirus and Fowlmouthvirus) each with one new species (Caudovirales: Siphoviridae https://ictv.global/ictv/proposals/2020.040B.R.Cukevirus_ Fowlmouthvirus_Indlulamithivirus.zip.Phage.Canada@gmail. com

106. Adriaenssens EM, Tolstoy I, Turner D, Kropinski AM (2020) Create one new subfamily (Deejayvirinae) including three new genera (Caudovirales: Siphoviridae) https://ictv.global/ictv/ proposals/2020.041B.R.Deejayvirinae.zip.Phage.Canada@ gmail.com

107. Caruso SM, deCarvalho TN, Erill I, Gill J, Gillis A (2020) Create two new genera (Deltatectivirus and Epsilontectivirus) including three new species (Kalamavirales: Tectiviridae) https://ictv. global/ictv/proposals/2020.042B.R.Deltatectivirus_Epsilontec tivirus.zip.scaruso@umbc.edu

108. Adriaenssens EM, Tolstoy I, Turner D, Kropinski AM (2020) Create 29 new species in two existing genera (Caudovirales: Demerecviridae) https://ictv.global/ictv/proposals/2020.043B.R. Demerecviridae_new_species.zip.Phage.Canada@gmail.com

109. Wittmann J, Adriaenssens EM, Kropinski AM (2020) Create one new genus (Dendoorenvirus) including one new species (Caudovirales: Schitoviridae) https://ictv.global/ictv/proposals/2020. 044B.R.Dendoorenvirus.zip. Johannes.Wittmann@dsmz.de

110. Adriaenssens EM, Tolstoy I, Kropinski AM, Barylski J, Gillis A (2020) Create one new genus (Deurplevirus) including one new species (Caudovirales: Siphoviridae) https:/ictv.global/ictv/ proposals/2020.045B.R.Deurplevirus.zip. Annika.gillis@gmail. com

111. Sadunishvili T, Kvesitadze G, Kropinski AM, Adriaenssens EM, Truncaitè L, Šimoliūnas E (2020) Create one new genus (Dibbivirus) including three new species (Caudovirales: Myoviridae) https://ictv.global/ictv/proposals/2020.046B.R.Dibbivirus.zip. Phage.Canada@gmail.com

112. Kropinski AM, Adriaenssens EM, Barylski J (2020) Create one new genus (Donellivirus) to include the species Bacillus virus 
G (Caudovirales: Myoviridae) https://ictv.global/ictv/proposals/ 2020.047B.R.Donellivirus.zip. Jakub.Barylski@ gmail.com

113. Adriaenssens EM, Tolstoy I, Moraru C Kropinski AM (2020) Create five new genera (Caudovirales: Drexlerviridae) https:// ictv.global/ictv/proposals/2020.048B.R.Drexlerviridae_new_ genera.zip.Phage.Canada@gmail.com

114. Adriaenssens EM, Tolstoy I, Turner D, Kropinski AM (2020) Create one new species in the genus Ruthyvirus (Caudovirales: Siphoviridae) https://ictv.global/ictv/proposals/2020.049B.R. Dumpstervirus.zip.Phage.Canada@gmail.com

115. Wittmann J, Adriaenssens EM, Kropinski AM (2020) Create one new genus (Eceepunavirus) including one new species (Caudovirales: Schitoviridae) https://ictv.global/ictv/proposals/2020. 050B.R.Eceepunavirus.zip.Johannes.Wittmann@dsmz.de

116. Adriaenssens EM, Tolstoy I, Turner D, Kropinski AM (2020) Create one new subfamily (Eekayvirinae) including two new genera (Caudovirales: Podoviridae) https://ictv.global/ictv/propo sals/2020.051B.R.Eekayvirinae.zip. Phage.Canada@gmail.com

117. Kropinski AM, Tolstoy I, Adriaenssens EM, Barylski J (2020) Create one new genus (Elmenteitavirus) including one new species (Caudovirales: Myoviridae) https://ictv.global/ictv/propo sals/2020.052B.R.Elmenteitavirus.zip. Jakub.Barylski@gmail. com

118. Kropinski AM, Tolstoy I, Turner D, Adriaenssens EM (2020) Create one new subfamily (Emmerichvirinae) including two new genera (Ishigurovirus and Ceceduovirus) (Caudovirales: Myoviridae) https://ictv.global/ictv/proposals/2020.053B.R.Emmer ichvirinae.zip. Phage.Canada@gmail.com

119. Adriaenssens EM, Tolstoy I, Kropinski AM, Moraru C, Wittmann J (2020) Create one subfamily (Enquatrovirinae) including three new genera (Caudovirales: Schitoviridae) https://ictv. global/ictv/proposals/2020.054B.R.Enquatrovirinae.zip. jow12@ dsmz.de

120. Kirchberger PC, Ochman H (2020) Create one new genus (Enterogokushovirus) in the subfamily Gokushovirinae (Microviridae) https://ictv.global/ictv/proposals/2020.055B.R.Enterogokushovi rus.zip.pkirchberger@utexas.edu

121. Adriaenssens EM, Tolstoy I, Kropinski AM, Moraru C, Wittmann J (2020) Create one new subfamily (Erskinevirinae) including two genera (Caudovirales: Podoviridae) https://ictv. global/ictv/proposals/2020.056B.R.Erskinevirinae.zip. jow12@ dsmz.de

122. Adriaenssens EM, Tolstoy I, Moraru C, Kropinski AM, Tsourkas PK, Barylski J (2020) Create 12 new species in the genus Fernvirus (formerly Sitaravirus) (Caudovirales: Siphoviridae) https://ictv.global/ictv/proposals/2020.057B.R.Fernvirus_12nsp. zip. Jakub.Barylski@gmail.com

123. Nilsson E, Holmfeldt $K$ (2020) Create six new genera including five new species in the genus Muminvirus, six new species in the genus Lillamyvirus, one new species in the genus Hattifnattvirus, two new species in the genus Pippivirus, one new species in the genus Tantvirus and one new species in the genus Labanvirus (Caudovirales) https://ictv.global/ictv/proposals/2020.058B.R. Flavobacterium_phages.zip. karin.holmfeldt@lnu.se

124. Adriaenssens EM, Tolstoy I, Moraru C, Turner D, Lueder M, Mahony J, Neve H, Moineau S, Kropinski AM (2020) Create one new genus (Fremauxvirus) including two new species (Caudovirales: Siphoviridae) https://ictv.global/ictv/proposals/2020. 059B.R.Fremauxvirus.zip. Phage.Canada@gmail.com

125. Adriaenssens EM, Tolstoy I, Kropinski AM, Jang HB, Sullivan MB, Moraru C, Wittmann J (2020) Create one new subfamily (Fuhrmanvirinae) including two new genera (Caudovirales: Schitoviridae) https://ictv.global/ictv/proposals/2020.060B.R. Fuhrmanvirinae.zip. jow12@dsmz.de

126. Kropinski AM, Turner D, Adriaenssens EM (2020) Create one new genus (Fukuivirus) including one new species and one existing species (Microcystis virus Ma-LMM01) (Caudovirales: Myoviridae) https://ictv.global/ictv/proposals/2020. 061B.R.Fukuivirus.zip.Phage.Canada@gmail.com

127. Adriaenssens EM, Tolstoy I, Turner D, Kropinski AM (2020) Create one new genus (Gilsonvirus) including two new species (Caudovirales: Siphoviridae) https://ictv.global/ictv/proposals/ 2020.062B.R.Gilsonvirus.zip. Phage.Canada@gmail.com

128. Krupovic M, Baquero DP, Prangishvili D (2020) Rename the genus Alphaglobulovirus and create two new species (Globuloviridae) https://ictv.global/ictv/proposals/2020.063B.R.Globu loviridae.zip.mart.krupovic@ pasteur.fr

129. Adriaenssens EM, Tolstoy I, Kropinski AM, Tsourkas PK (2020) Create one new subfamily (Gochnauervirinae) including four genera (Caudovirales: Siphoviridae) https://ictv. global/ictv/proposals/2020.064B.R.Gochnauervirinae.zip. Phage.Canada@gmail.com

130. Adriaenssens EM, Tolstoy I, Kropinski AM, Moraru C (2020) Create one new subfamily (Gorgonvirinae) including two genera (Caudovirales: Myoviridae) https://ictv.global/ictv/propo sals/2020.065B.R.Gorgonvirinae.zip. Phage.Canada@gmail. com

131. Adriaenssens EM, Tolstoy I, Łobocka M, Moraru C, Barylski J, Tong Y, Kropinski AM (2020) Create one new family (Guelinviridae) of Clostridium phages including one new subfamily, four new genera and nine new species (Caudovirales) https:// ictv.global/ictv/proposals/2020.066B.R.Guelinviridae.zip. Phage. Canada@gmail.com

132. Adriaenssens EM, Tolstoy I, Kropinski AM, Barylski J (2020) Create one new subfamily (Gutmannvirinae) including two new genera and three new species (Caudovirales: Siphoviridae) https://ictv.global/ictv/proposals/2020.067B.R.Gutmannvirinae. zip. Jakub.Barylski@gmail.com

133. Adriaenssens EM, Tolstoy I, Moraru C, Kropinski AM, Tsourkas PK, Barylski J (2020) Rename the genus Halcyonevirus (formerly Trippvirus) and create four new species (Caudovirales: Siphoviridae) https://ictv.global/ictv/proposals/2020.068B.R. Halcyonevirus.zip. Jakub.Barylski@gmail.com

134. Adriaenssens EM, Tolstoy I, Moraru C, Kropinski AM (2020) Create 13 new species in the genus Hanrivervirus (Caudovirales: Drexlerviridae) https://ictv.global/ictv/proposals/2020.069B.R. Hanrivervirus.zip. Phage.Canada@gmail.com

135. Lehman SM, Petit MA, Lossouarn J, Tong Y, Tolstoy I, Adriaenssens E (2020) Promote the genus Hendrixvirus to the rank of subfamily (Hendrixvirinae) and establish nine new genera (Caudovirales: Siphoviridae) https://ictv.global/ictv/proposals/ 2020.070B.R.Hendrixvirinae.zip.susan.lehman@fda.hhs.gov

136. Adriaenssens EM, Tolstoy I, Turner D, Kropinski AM (2020) Create one new genus (Henunavirus) including two new species in the subfamily Vequintavirinae (Caudovirales: Myoviridae) https://ictv.global/ictv/proposals/2020.071B.R.Henunavirus.zip. Phage.Canada@gmail.com

137. Adriaenssens EM, Tolstoy I, Kropinski AM, Moraru C, Hertel R, Lehman SM, Barylski J (2020) Create 13 new genera and move two genera (Caudovirales: Herelleviridae) https://ictv.global/ ictv/proposals/2020.072B.R.Herelleviridae.zip. Jakub.Barylski@ gmail.com

138. Adriaenssens EM, Tolstoy I, Turner D, Kropinski AM (2020) Create one new genus (Hnatkovirus) including one new species (Caudovirales: Siphoviridae) https://ictv.global/ictv/proposals/ 2020.073B.R.Hnatkovirus.zip.Phage.Canada@gmail.com

139. Adriaenssens EM, Tolstoy I, Kropinski AM, Barylski J (2020) Create one new genus (Hubeivirus) including two new species (Caudovirales: Siphoviridae) https://ictv.global/ictv/proposals/ 2020.074B.R.Hubeivirus.zip. Jakub.Barylski@gmail.com

140. Adriaenssens EM, Tolstoy I, Kropinski AM, Moraru C, Wittmann J (2020) Create one new genus (Huelvavirus) including 
one new species (Caudovirales: Schitoviridae) https://ictv.global/ ictv/proposals/2020.075B.R.Huelvavirus.zip. jow12@dsmz.de

141. Adriaenssens EM, Tolstoy I, Kropinski AM, Moraru C, Wittmann J (2020) Create one new subfamily (Humphriesvirinae) including three existing genera (Caudovirales: Schitoviridae) https://ictv.global/ictv/proposals/2020.076B.R.Humphriesvirinae.zip.jow12@dsmz.de

142. Adriaenssens EM, Tolstoy I, Moraru C Kropinski AM (2020) Create three new species in the genus Ilzatvirus (Caudovirales: Siphoviridae) https://ictv.global/ictv/proposals/2020.077B.R. Ilzatvirus.zip.Phage.Canada@gmail.com

143. Adriaenssens EM, Tolstoy I, Kropinski AM, Barylski J (2020) Create one new genus (Jarrellvirus) including one new species (Caudovirales: Siphoviridae) https://ictv.global/ictv/proposals/ 2020.078B.R.Jarrellvirus.zip. Jakub.Barylski@gmail.com

144. Adriaenssens EM,Tolstoy I, Kropinski AM, Morozova V, Barylski J (2020) Create one new genus (Kamchatkavirus) including one new species (Caudovirales: Siphoviridae) https://ictv. global/ictv/proposals/2020.079B.R.Kamchatkavirus.zip. Jakub. Barylski@gmail.com

145. Kropinski AM, Adriaenssens EM, Turner D (2020) Create one new genus (Kanagawavirus) including two new species (Caudovirales: Myoviridae) https://ictv.global/ictv/proposals/2020. 080B.R.Kanagawavirus.zip.Phage.Canada@gmail.com

146. Adriaenssens EM, Tolstoy I, Turner D, Kropinski AM (2020) Create one new genus (Karimacvirus) including five new species (Caudovirales: Siphoviridae) https://ictv.global/ictv/proposals/ 2020.081B.R.Karimacvirus.zip. Phage.Canada@gmail.com

147. Kropinski AM, Adriaenssens EM, Neve H, Franz CMAP, Sprotte S (2020) Create one new genus (Knuthellervirus) including one new species (Caudovirales: Siphoviridae) https://ictv.global/ictv/ proposals/2020.082B.R.Knuthellervirus.zip. Phage.Canada@ gmail.com

148. Kropinski AM, Turner D, Adriaenssens EM (2020) Create one new genus (Kozyakovvirus) including one new species (Caudovirales: Podoviridae) https://ictv.global/ictv/proposals/2020. 083B.R.Kozyakovvirus.zip.Phage.Canada@gmail.com

149. Adriaenssens EM, Tolstoy I, Moraru C, Kropinski AM (2020) Create one new genus (Kuleanavirus) including one new species (Caudovirales: Siphoviridae) https://ictv.global/ictv/proposals/ 2020.084B.R.Kuleanavirus.zip.Phage.Canada@gmail.com

150. Adriaenssens EM, Tolstoy I, Kropinski AM (2020) Create one new genus (Kungbxnavirus) including one new species (Caudovirales: Myoviridae) https://ictv.global/ictv/proposals/2020. 085B.R.Kungbxnavirus.zip.Phage.Canada@gmail.com

151. Adriaenssens EM, Tolstoy I, Turner D, Kropinski AM (2020) Create one new genus (Kylevirus) including one new species (Caudovirales: Myoviridae) https://ictv.global/ictv/proposals/ 2020.086B.R.Kylevirus.zip.Phage.Canada@gmail.com

152. Kabwe M, Petrovski S, Tucci J, Adriaenssens EM, Tolstoy I, Kropinski AM (2020) Create one new genus (Lahexavirus) including four new species (Caudovirales: Podoviridae) https://ictv.global/ ictv/proposals/2020.087B.R.Lahexavirus.zip.Phage.Canada@ gmail.com

153. Adriaenssens EM, Tolstoy I, Turner D, Kropinski AM (2020) Create one new genus (Lambovirus) including five new species (Caudovirales: Siphoviridae) https://ictv.global/ictv/proposals/ 2020.088B.R.Lambovirus.zip.Phage.Canada@gmail.com

154. Kropinski AM, Tolstoy I, Turner D, Adriaenssens EM (2020) Create 12 new genera including 17 new species for Lactobacillus viruses (Caudovirales: Siphoviridae) https://ictv.global/ictv/ proposals/2020.089B.R.Lactobacillus_siphoviruses.zip. Phage. Canada@gmail.com

155. Adriaenssens EM, Tolstoy I, Moraru C, Kropinski AM (2020) Create three new species in the genus Laroyevirus (Caudovirales:
Siphoviridae) https://ictv.global/ictv/proposals/2020.090B.R. Laroyevirus.zip.Phage.Canada@gmail.com

156. Adriaenssens EM, Tolstoy I, Kropinski AM (2020) Create one new genus (Lastavirus) including two new species (Caudovirales: Podoviridae) https://ictv.global/ictv/proposals/2020. 091B.R.Lastavirus.zip.Phage.Canada@gmail.com

157. Kropinski AM, Tolstoy I, Adriaenssens EM, Kabwe M, Tucci J (2020) Create one new genus (Latrobevirus) including one new species (Caudovirales: Siphoviridae) https://ictv.global/ictv/ proposals/2020.092B.R.Latrobevirus.zip.Phage.Canada@gmail. com

158. Adriaenssens EM, Tolstoy I, Turner D, Kropinski AM (2020) Create one new genus (Leicestervirus) including three new species (Caudovirales: Siphoviridae) https://ictv.global/ictv/propo sals/2020.093B.R.Leicestervirus.zip.Phage.Canada@gmail.com

159. Adriaenssens EM, Tolstoy I, Moraru C Kropinski AM (2020) Create one new genus (Seongbukvirus) including one new species and create four new species of Leuconostoc phages in two existing genera (Caudovirales: Siphoviridae) https://ictv.global/ ictv/proposals/2020.094B.R.Leuconostoc_siphoviruses.zip. Phage.Canada@gmail.com

160. Callanan J, Stockdale SR, Adriaenssens EM, Kuhn JH, Rumnieks J, Shkoporov A, Draper LA, Ross RP, Hill C (2020) Rename one class (Leviviricetes - formerly Allassoviricetes), rename one order (Norzivirales - formerly Levivirales), create one new order (Timlovirales), and expand the class to a total of six families, 420 genera and 883 species https://ictv.global/ictv/proposals/2020. 095B.R.Leviviricetes.zip.c.hill@ucc.ie

161. Truncaitè L, Kropinski AM, Adriaenssens EM, Šimoliūnas E (2020) Create one new genus (Lietduovirus) including one new species (Caudovirales: Myoviridae) https://ictv.global/ictv/propo sals/2020.096B.R.Lietduovirus.zip.Phage.Canada@gmail.com

162. Wittmann J, Adriaenssens EM, Kropinski AM (2020) Create one new genus (Littlefixvirus) containing one new species (Caudovirales: Schitoviridae) https://ictv.global/ictv/proposals/2020. 097B.R.Littlefixvirus.zip.Johannes.Wittmann@dsmz.de

163. Kropinski AM, Tolstoy I, Turner D, Adriaenssens EM (2020) Create one new genus (Marfavirus) including two new species (Caudovirales: Myoviridae) https://ictv.global/ictv/proposals/ 2020.098B.R.Marfavirus.zip. Phage.Canada@gmail.com

164. Adriaenssens EM, Tolstoy I, Moraru C, Kropinski AM (2020) Create one new species in the genus Marthavirus (Caudovirales: Myoviridae) https://ictv.global/ictv/proposals/2020.099B.R. Marthavirus.zip.Phage.Canada@gmail.com

165. Oksanen HM, Krupovic M, Jalasvuori M (2020) Create one new family (Matsushitaviridae) including one renamed genus (Hukuchivirus - formerly Gammasphaerolipovirus) moved from the family Sphaerolipoviridae (Halopanivirales) https://ictv.global/ ictv/proposals/2020.100B.R.Matsushitaviridae.zip. matti.jalasvuori@jyu.fi

166. Adriaenssens EM, Tolstoy I, Kropinski AM, Ramsey J (2020) Create one new genus (Menderavirus) including three new species (Caudovirales: Myoviridae) https://ictv.global/ictv/propo sals/2020.101B.R.Menderavirus.zip.Phage.Canada@gmail.com

167. Adriaenssens EM, Tolstoy I, Kropinski AM, Moraru C, Wittmann J (2020) Create one new subfamily (Migulavirinae) including two existing genera (Caudovirales: Schitoviridae) https://ictv. global/ictv/proposals/2020.102B.R.Migulavirinae.zip. jow12@ dsmz.de

168. Adriaenssens EM, Tolstoy I, Kropinski AM Ramsey J (2020) Create one new genus (Moabitevirus) including two new species (Caudovirales: Myoviridae) https://ictv.global/ictv/propo sals/2020.103B.R.Moabitevirus.zip.Phage.Canada@gmail.com

169. Adriaenssens EM, Tolstoy I, Kropinski AM (2020) Create one new genus (Moturavirus) including one new species 
(Caudovirales: Myoviridae) https://ictv.global/ictv/proposals/ 2020.104B.R.Moturavirus.zip.Phage.Canada@gmail.com

170. Adriaenssens EM, Tolstoy I, Moraru C, Kropinski AM (2020) Create one new genus (Mufasoctovirus) including one new species (Caudovirales: Siphoviridae) https://ictv.global/ictv/propo sals/2020.105B.R.Mufasoctovirus.zip.Phage.Canada@gmail. com

171. Adriaenssens EM, Tolstoy I, Kropinski AM, Ramsey J (2020) Create one new genus (Muldoonvirus) including two new species (Caudovirales: Myoviridae) https://ictv.global/ictv/propo sals/2020.106B.R.Muldoonvirus.zip.Phage.Canada@gmail. com

172. Adriaenssens EM, Tolstoy I, Turner D, Kropinski AM (2020) Create one new genus (Mydovirus) including six new species in the subfamily Vequintavirinae (Caudovirales: Myoviridae) https://ictv.global/ictv/proposals/2020.107B.R.Mydovirus.zip. Phage.Canada@gmail.com

173. Gonzalez-Serrano R, Dunne M, Rosselli R, Martin-Cuadrado A-B, Grosboillot V, Zinsli LV, Roda-Garcia JJ, Loessner MJ, Rodriguez-Valera F (2020) Create one new genus (Myoalterovirus) including one new species (Caudovirales: Myoviridae) https://ictv.global/ictv/proposals/2020.108B.R.Myoalterovirus. zip.rafael.gonzalezs@umh.es

174. Adriaenssens EM, Tolstoy I, Turner D, Kropinski AM (2020) Create one new genus (Myosmarvirus) including two new species (Caudovirales: Myoviridae) https://ictv.global/ictv/propo sals/2020.109B.R.Myosmarvirus.zip. Phage.Canada@gmail.com

175. Adriaenssens EM, Tolstoy I, Turner D, Lueder M, Moineau S, Kropinski AM (2020) Create one new genus (Nevevirus) including four new species (Caudovirales: Siphoviridae) https://ictv. global/ictv/proposals/2020.110B.R.Nevevirus.zip. Phage.Canada@gmail.com

176. Kropinski AM, Tolstoy I, Turner D, Adriaenssens EM (2020) Create one new genus (Nylescharonvirus) including two new species (Caudovirales: Myoviridae) https://ictv.global/ictv/propo sals/2020.111B.R.Nylescharonvirus.zip.Phage.Canada@gmail. com

177. Adriaenssens EM, Tolstoy I, Kropinski AM, Moraru C, Wittmann J (2020) Create one new genus (Pacinivirus) including two new species (Caudovirales: Schitoviridae) https://ictv.global/ictv/ proposals/2020.112B.R.Pacinivirus.zip.jow12@dsmz.de

178. Adriaenssens EM, Tolstoy I, Kropinski AM Ramsey J (2020) Create one new genus (Parlovirus) including one new species (Caudovirales: Podoviridae) https://ictv.global/ictv/proposals/ 2020.113B.R.Parlovirus.zip.Phage.Canada@gmail.com

179. Roux S, Krupovic M (2020) Create one new family (Paulinoviridae) including two genera moved from the family Inoviridae (Tubulavirales) https://ictv.global/ictv/proposals/2020.114B.R. Paulinoviridae.zip.siroux1@gmail.com

180. Adriaenssens EM, Tolstoy I, Turner D, Kropinski AM (2020) Abolish one genus (Pbilvirus) and rename one species (Mycobacterium virus Plot) (Caudovirales: Siphoviridae) https://ictv. global/ictv/proposals/2020.115B.R.Pbilvirus.zip. Phage.Canada@gmail.com

181. Adriaenssens EM, Tolstoy I, Turner D, Kropinski AM (2020) Create one new genus (Bcepfunavirus) and create nine new species in the genus Pbunavirus (Caudovirales: Myoviridae) https:// ictv.global/ictv/proposals/2020.116B.R.Pbunavirus.zip. Phage. Canada@gmail.com

182. van Zyl LJ, Lueder MR, Bishop-Lilly KA, Turner D, Adriaenssens EM, Kropinski AM (2020) Create five new genera and 29 new species in the subfamily Peduovirinae (Caudovirales: Myoviridae) https://ictv.global/ictv/proposals/2020.117B.R.Peduo viruses.zip. lvanzyl@uwc.ac.za

183. Kropinski AM, Adriaenssens EM, Tolstoy I, Barylski J (2020) Create one new genus (Pimunavirus) including one new species
(Caudovirales: Myoviridae) https://ictv.global/ictv/proposals/ 2020.118B.R.Pemunavirus.zip. Jakub.Barylski@gmail.com

184. Adriaenssens EM, Tolstoy I, Turner D, Kropinski AM (2020) Create one new genus (Plateaulakevirus) including four new species (Caudovirales: Myoviridae) https://ictv.global/ictv/propo sals/2020.119B.R.Plateaulakevirus.zip.Phage.Canada@gmail. com

185. Adriaenssens EM, Tolstoy I, Kropinski AM, Moraru C, Wittmann J (2020) Create one new genus (Pokkenvirus) including one new species (Caudovirales: Schitoviridae) https://ictv.global/ ictv/proposals/2020.120B.R.Pokkenvirus.zip. jow12@dsmz.de

186. Adriaenssens EM, Tolstoy I, Kropinski AM, Moraru C, Wittmann J (2020) Create one new genus (Pollockvirus) including one species moved from the genus Ithacavirus (Caudovirales: Schitoviridae) https://ictv.global/ictv/proposals/2020.121B.R. Pollockvirus.zip. jow12@dsmz.de

187. Adriaenssens EM, Tolstoy I, Kropinski AM, Jang HB, Sullivan MB, Moraru C, Wittmann J (2020) Create one new subfamily (Pontosvirinae) including three new genera (Caudovirales: Schitoviridae) https://ictv.global/ictv/proposals/2020.122B.R.Ponto svirinae.zip. jow12@dsmz.de

188. Wittmann J, Adriaenssens EM, Kropinski AM (2020) Create one new genus (Presleyvirus) including one new species (Caudovirales: Schitoviridae) https://ictv.global/ictv/proposals/2020. 123B.R.Presleyvirus.zip. Johannes.Wittmann@dsmz.de

189. Adriaenssens EM, Tolstoy I, Kropinski AM Ramsey J (2020) Create one new genus (Privateervirus) including two new species (Caudovirales: Podoviridae) https://ictv.global/ictv/propo sals/2020.124B.R.Privateervirus.zip.Phage.Canada@gmail.com

190. Kropinski AM, Adriaenssens EM, Moraru C (2020) Create one new genus (Pseudotevenvirus) including nine species in the subfamily Tevenvirinae (Caudovirales: Myoviridae) https://ictv. global/ictv/proposals/2020.125B.R.Pseudotevenvirus.zip. Phage. Canada@gmail.com

191. Adriaenssens EM, Tolstoy I, Kropinski AM, Moraru C, Wittmann J (2020) Create one new genus (Pylasvirus) including two new species (Caudovirales: Schitoviridae) https://ictv.global/ictv/ proposals/2020.126B.R.Pylasvirus.zip. jow12@dsmz.de

192. Adriaenssens EM, Tolstoy I, Turner D, Lueder M, Mahony J, Neve H, Moineau S, Kropinski AM (2020) Create one new genus (Questintvirus) including one new species (Caudovirales: Siphoviridae) https://ictv.global/ictv/proposals/2020.127B.R.Quest intvirus.zip.Phage.Canada@gmail.com

193. Ma R, Zhang R (2020) Create one new subfamily (Queuovirinae) including one new genus (Amoyvirus) and three existing genera (Caudovirales: Siphoviridae) https://ictv.global/ictv/proposals/ 2020.128B.R.Queuovirinae.zip. maruijie@stu.xmu.edu.cn

194. Adriaenssens EM, Tolstoy I, Moraru C, Kropinski AM, Kaliniene L (2020) Rename one genus (Klausavirus - formerly Radnorvirus) and create three new species (Caudovirales: Myoviridae) https://ictv.global/ictv/proposals/2020.129B.R.Radnorvirus_ rename.zip.laura.kaliniene@bchi.vu.lt

195. Torres-Barceló C, Trotereau A (2020) Create seven new genera and 13 new species of Ralstonia phages (Caudovirales) https:// ictv.global/ictv/proposals/2020.130B.R.Ralstonia_phages.zip. clara.torresbarcelo@inrae.fr

196. Adriaenssens EM, Tolstoy I, Turner D, Holmfeldt K, Šulčius S, Kropinski AM (2020) Create one new genus (Ravarandavirus) including three new species (Caudovirales: Siphoviridae) https://ictv.global/ictv/proposals/2020.131B.R.Ravarandavirus. zip.Phage.Canada@gmail.com

197. Kropinski AM, Turner D, Adriaenssens EM (2020) Create two new species and move the species Rhodococcus virus RGL3 to the genus Rerduovirus (Caudovirales: Siphoviridae) https://ictv. global/ictv/proposals/2020.132B.R.Rerduovirus.zip. Phage.Canada@gmail.com 
198. Adriaenssens EM, Tolstoy I, Kropinski AM, Moraru C, Wittmann J (2020) Create one new subfamily (Rhodovirinae) including seven genera (Caudovirales: Schitoviridae) https://ictv. global/ictv/proposals/2020.133B.R.Rhodovirinae.zip. jow12@ dsmz.de

199. Wittmann J, Adriaenssens EM, Kropinski AM (2020) Create one new genus (Riverridervirus) including one new species (Caudovirales: Schitoviridae) https://ictv.global/ictv/proposals/2020. 134B.R.Riverridervirus.zip. Johannes.Wittmann@ dsmz.de

200. Adriaenssens EM, Tolstoy I, Kropinski AM, Łobocka M (2020) Create one new genus (Rockefellervirus) including six species (Caudovirales: Siphoviridae) https://ictv.global/ictv/proposals/ 2020.135B.R.Rockefellervirus.zip. lobocka@ibb.waw.pl

201. Kropinski AM, Tolstoy I, Adriaenssens EM, Barylski J (2020) Create one new genus (Rockvillevirus) including one new species (Caudovirales: Siphoviridae) https://ictv.global/ictv/proposals/ 2020.136B.R.Rockvillevirus.zip. Jakub.Barylski@gmail.com

202. Adriaenssens EM, Tolstoy I, Turner D, Kropinski AM (2020) Create one new genus (Ronodorvirus) including two new species (Caudovirales: Myoviridae) https://ictv.global/ictv/propo sals/2020.137B.R.Ronodorvirus.zip.Phage.Canada@gmail.com

203. Adriaenssens EM, Tolstoy I, Turner D, Kropinski AM (2020) Create four new species in the genus Rosemountvirus (Caudovirales: Myoviridae) https://ictv.global/ictv/proposals/2020. 138B.R.Rosemountvirus.zip.Phage.Canada@gmail.com

204. Adriaenssens EM, Tolstoy I, Kropinski AM, Moraru C, Wittmann J (2020) Create one new subfamily (Rothmandenesvirinae) including four genera (Caudovirales: Schitoviridae) https://ictv. global/ictv/proposals/2020.139B.R.Rothmandenesvirinae.zip. jow12@dsmz.de

205. Adriaenssens EM, Tolstoy I, Moraru C, Barylski J, Tong Y, Kropinski AM, Łobocka M (2020) Create one new family (Rountreeviridae) including two subfamilies and six genera of predominantly Staphylococcus and Enterococcus phages (Caudovirales) https://ictv.global/ictv/proposals/2020.140B.R.Rountreeviridae. zip.Phage.Canada@gmail.com

206. Krupovic M, Baquero DP, Prangishvili D (2020) Create six new genera and 14 new species (Ligamenvirales: Rudiviridae) https:// ictv.global/ictv/proposals/2020.141B.R.Rudiviridae.zip. mart. krupovic@pasteur.fr

207. Kropinski AM, Tolstoy I, Turner D, Adriaenssens EM (2020) Create one new genus (Saintgironsvirus) including one new species (Caudovirales: Myoviridae) https://ictv.global/ictv/propo sals/2020.142B.R.Saintgironsvirus.zip.Phage.Canada@gmail. com

208. Adriaenssens EM, Tolstoy I, Moraru C, Kropinski AM, Barylski J (2020) Create two new genera in the family Podoviridae and create one new family (Salasmaviridae) including one moved subfamily (Picovirinae), two new subfamilies (Northropvirinae and Tatarstanvirinae), and nine new genera (Caudovirales) https://ictv.global/ictv/proposals/2020.143B.R.Salasmaviridae. zip. Jakub.Barylski@gmail.com

209. Adriaenssens EM, Tolstoy I, Moraru C, Turner D, Lueder M, Moineau S, Kropinski AM (2020) Create one new genus (Sandinevirus) including new one species (Caudovirales: Siphoviridae) https://ictv.global/ictv/proposals/2020.144B.R.Sandinevirus.zip. Phage.Canada@gmail.com

210. Adriaenssens EM, Tolstoy I, Kropinski AM (2020) Create one new genus (Sarumanvirus) including two new species (Caudovirales: Myoviridae) https://ictv.global/ictv/proposals/2020. 145B.R.Sarumanvirus.zip.Phage.Canada@gmail.com

211. Adriaenssens EM, Tolstoy I, Kropinski AM, Moraru C, Wittmann J (2020) Create one new family (Schitoviridae) including eight existing subfamilies and 40 existing genera (Caudovirales: Schitoviridae) https://ictv.global/ictv/proposals/2020.146B.R. Schitoviridae.zip. jow12@dsmz.de
212. Kropinski AM, Adriaenssens EM (2020) Create one new genus (Sendosyvirus) including two species (Caudovirales: Podoviridae) https://ictv.global/ictv/proposals/2020.147B.R. Sendosyvirus.zip.Phage.Canada@gmail.com

213. Lehman SM, Petit MA, Lossouarn J, Tong Y, Tolstoy I, Adriaenssens E (2020) Create ten new species in the subfamily Sepvirinae (Caudovirales: Podoviridae) https://ictv.global/ ictv/proposals/2020.148B.R.Sepvirinae.zip.susan.lehman@ fda.hhs.gov

214. Kropinski AM, Turner D, Adriaenssens EM (2020) Create one new genus (Shandongvirus) including one new species (Caudovirales: Myoviridae) https://ictv.global/ictv/proposals/2020. 149B.R.Shandongvirus.zip.Phage.Canada@gmail.com

215. Adriaenssens EM, Tolstoy I, Kropinski AM (2020) Create one new genus (Shirahamavirus) including one new species (Caudovirales: Myoviridae) https://ictv.global/ictv/proposals/2020. 150B.R.Shirahamavirus.zip.Phage.Canada@gmail.com

216. Liu Y, Du S, Chen X, Krupovic M (2020) Create one new family (Simuloviridae) including one genus (Yingchengvirus - formerly Betasphaerolipovirus) moved from the family Sphaerolipoviridae (Halopanivirales) https://ictv.global/ictv/proposals/2020. 151B.R.Simuloviridae.zip. mart.krupovic@ pasteur.fr

217. Kropinski AM, Tolstoy I, Adriaenssens EM, Barylski J, Piligrimova EG, Kazantseva OA, Nikulin NA, Shadrin AM (2020) Create one new subfamily (Skryabinvirinae) including two new genera, each with one new species (Caudovirales: Siphoviridae) https://ictv.global/ictv/proposals/2020.152B.R.Skryabinvirinae. zip. Jakub.Barylski@gmail.com

218. Adriaenssens EM, Tolstoy I, Moraru C, Turner D, Lueder M, Moineau S, Kropinski AM (2020) Create 80 new species in the genus Skunavirus (Caudovirales: Siphoviridae) https://ictv. global/ictv/proposals/2020.153B.R.Skunavirus.zip. Phage.Canada@gmail.com

219. Adriaenssens EM, Tolstoy I, Turner D, Kropinski AM (2020) Create one new genus (Sleepyheadvirus) including one new species (Caudovirales: Siphoviridae) https://ictv.global/ictv/propo sals/2020.154B.R.Sleepyheadvirus.zip.Phage.Canada@gmail. com

220. Kropinski AM, Tolstoy I, Turner D, Adriaenssens EM (2020) Create one new genus (Sozzivirus) including three new species (Caudovirales: Siphoviridae) https://ictv.global/ictv/proposals/ 2020.155B.R.Sozzivirus.zip.Phage.Canada@gmail.com

221. Adriaenssens EM, Tolstoy I, Turner D, Kropinski AM (2020) Create one new genus (Sparkyvirus) including one new species (Caudovirales: Siphoviridae) https://ictv.global/ictv/proposals/ 2020.156B.R.Sparkyvirus.zip.Phage.Canada@gmail.com

222. Kropinski AM, Tolstoy I, Adriaenssens EM, Barylski J (2020) Create one new genus (Spizizenvirus) including one new species (Caudovirales: Siphoviridae) https://ictv.global/ictv/proposals/ 2020.157B.R.Spizizenvirus.zip. Jakub.Barylski@gmail.com

223. Adriaenssens EM, Tolstoy I, Turner D, Kropinski AM (2020) Create one new genus (Squirtyvirus) including one new species (Caudovirales: Siphoviridae) https://ictv.global/ictv/proposals/ 2020.158B.R.Squirtyvirus.zip.Phage.Canada@gmail.com

224. Adriaenssens EM, Tolstoy I, Turner D, Kropinski AM (2020) Create four new species in the genus Samwavirus (Caudovirales: Siphoviridae) https://ictv.global/ictv/proposals/2020.159B.R. Samwavirus_new_species.zip. Phage.Canada@gmail.com

225. Kropinski AM, Adriaenssens EM, Barylski J (2020) Create one new genus (Takahashivirus) including one existing species (Bacillus virus PBS1) (Caudovirales: Myoviridae) https://ictv. global/ictv/proposals/2020.160B.R.Takahashivirus.zip. Jakub. Barylski@gmail.com

226. Adriaenssens EM, Tolstoy I, Kropinski AM Barylski J (2020) Create one new genus (Tandoganvirus) including one new species (Caudovirales: Siphoviridae) https://ictv.global/ictv/propo 
sals/2020.161B.R.Tandoganvirus.zip. Jakub.Barylski@gmail. com

227. Adriaenssens EM, Tolstoy I, Moraru C, Kropinski AM (2020) Create 53 new species, rename one species and move one species in the genus Tequatrovirus (Caudovirales: Myoviridae) https://ictv.global/ictv/proposals/2020.162B.R.Tequatrovirus. zip.Phage.Canada@gmail.com

228. Adriaenssens EM, Tolstoy I, Moraru C, Turner D, Lueder M, Neve H, Mahony J, Moineau S, Kropinski AM (2020) Create one new genus (Teubervirus) including five new species (Caudovirales: Siphoviridae) https://ictv.global/ictv/proposals/ 2020.163B.R.Teubervirus.zip. Phage.Canada@gmail.com

229. Adriaenssens EM, Tolstoy I, Turner D, Kropinski AM (2020) Create one new genus (Thetabobvirus) including three new species (Caudovirales: Siphoviridae) https://ictv.global/ictv/ proposals/2020.164B.R.Thetabobvirus.zip.Phage.Canada@ gmail.com

230. Adriaenssens EM, Tolstoy I, Moraru C, Kropinski AM (2020) Create one new subfamily (Trabyvirinae) including two new genera (Caudovirales: Siphoviridae) https://ictv.global/ictv/propo sals/2020.165B.R.Trabyvirinae.zip. Phage.Canada@gmail.com

231. Adriaenssens EM, Tolstoy I, Kropinski AM, Łobocka M (2020) Create 17 new species in the genus Triavirus (Caudovirales: Siphoviridae) https://ictv.global/ictv/proposals/2020.166B.R. Triavirus.zip. lobocka@ibb.waw.pl

232. Adriaenssens EM, Tolstoy I, Moraru C, Kropinski AM (2020) Create one new genus (Triplejayvirus) including one new species (Caudovirales: Siphoviridae) https://ictv.global/ictv/proposals/ 2020.167B.R.Triplejayvirus.zip. Phage.Canada@gmail.com

233. Krupovic M, Baquero DP, Prangishvili D (2020) Create one new genus (Betatristromavirus) including one existing species (Thermoproteus tenax virus 1 ) and create one new species in the genus Alphatristromavirus (Tristromaviridae) https://ictv. global/ictv/proposals/2020.168B.R.Tristromaviridae.zip. mart. krupovic@pasteur.fr

234. Adriaenssens EM, Tolstoy I, Moraru C, Kropinski AM (2020) Create four new species and abolish one species in the genus Tunavirus (Caudovirales: Drexlerviridae) https://ictv.global/ictv/ proposals/2020.169B.R.Tunavirus.zip.Phage.Canada@gmail. com

235. Adriaenssens EM, Tolstoy I, Kropinski AM Oliveira H, Turner D, Moraru C (2020) Create one new subfamily (Twarogvirinae) including five new genera (Caudovirales: Podoviridae) https:// ictv.global/ictv/proposals/2020.170B.R.Twarogvirinae.zip. Phage.Canada@gmail.com

236. Adriaenssens EM, Tolstoy I, Turner D, Lueder M, Nakai T, Kropinski AM (2020) Create one new genus (Uwajimavirus) including one new species (Caudovirales: Siphoviridae) https://ictv. global/ictv/proposals/2020.171B.R.Uwajimavirus.zip. Phage. Canada@gmail.com

237. Adriaenssens EM, Tolstoy I, Turner D, Lueder M, Moineau S, Mahony J, Kropinski AM (2020) Create one new genus (Vedamuthuvirus) including five new species (Caudovirales: Siphoviridae) https://ictv.global/ictv/proposals/2020.172B.R.Vedam uthuvirus.zip.Phage.Canada@gmail.com

238. Adriaenssens EM, Tolstoy I, Moraru C, Kropinski AM (2020) Create one new genus (Veterinaerplatzvirus) including one new species (Caudovirales: Drexlerviridae) https://ictv.global/ictv/ proposals/2020.173B.R.Veterinaerplatzvirus.zip. Phage.Canada@gmail.com

239. Adriaenssens EM, Tolstoy I, Moraru C, Kropinski AM (2020) Create one new genus (Vibakivirus) including one new species (Caudovirales: Myoviridae) https://ictv.global/ictv/proposals/ 2020.174B.R.Vibakivirus.zip.Phage.Canada@gmail.com

240. Wittmann J, Adriaenssens EM, Kropinski AM (2020) Create one new genus (Waedenswilvirus) including one new species
(Caudovirales: Schitoviridae) https://ictv.global/ictv/proposals/ 2020.175B.R.Waedenswilvirus.zip. Johannes.Wittmann@dsmz. de

241. Adriaenssens EM, Tolstoy I, Kropinski AM, Turner D, Łobocka M (2020) Create one new genus (Warsawvirus) including one new species (Caudovirales: Autographiviridae) https://ictv. global/ictv/proposals/2020.176B.R.Warsawvirus.zip.lobocka@ ibb.waw.pl

242. Adriaenssens EM, Tolstoy I, Moraru C, Kropinski AM (2020) Create eight new species and abolish one species in the genus Warwickvirus (Caudovirales: Drexlerviridae) https://ictv.global/ ictv/proposals/2020.177B.R.Warwickvirus.zip.Phage.Canada@ gmail.com

243. Kropinski AM, Tolstoy I, Adriaenssens EM, Barylski J (2020) Create one new genus (Waukeshavirus) including two new species (Caudovirales: Siphoviridae) https://ictv.global/ictv/propo sals/2020.178B.R.Waukeshavirus.zip. Jakub.Barylski@gmail. com

244. Adriaenssens EM, Tolstoy I, Moraru C, Kropinski AM (2020) Create 16 new species and abolish two species in the genus Webervirus (Caudovirales: Drexlerviridae) https://ictv.global/ ictv/proposals/2020.179B.R.Webervirus.zip. Phage.Canada@ gmail.com

245. Adriaenssens EM, Tolstoy I, Turner D, Kropinski AM (2020) Create one new genus (Whackvirus) including one new species (Caudovirales: Siphoviridae) https://ictv.global/ictv/proposals/ 2020.180B.R.Whackvirus.zip.Phage.Canada@gmail.com

246. Adriaenssens EM, Tolstoy I, Mahony J, Neve H, Moineau S, Kropinski AM (2020) Create one new genus (Whiteheadvirus) including one new species (Caudovirales: Siphoviridae) https:// ictv.global/ictv/proposals/2020.181B.R. Whiteheadvirus.zip. Phage.Canada@gmail.com

247. Kropinski AM, Tolstoy I, Adriaenssens EM, Turner D (2020) Create one new genus (Wumpquatrovirus) including one new species (Caudovirales: Podoviridae) https://ictv.global/ictv/ proposals/2020.182B.R.Wumpquatrovirus.zip.Dann2.Turner@ uwe.ac.uk

248. Kropinski AM, Tolstoy I, Adriaenssens EM, Turner D (2020) Create one new genus (Wumptrevirus) including two species (Caudovirales: Podoviridae) https://ictv.global/ictv/proposals/ 2020.183B.R.Wumptrevirus.zip. Dann2.Turner@uwe.ac.uk

249. Adriaenssens EM, Tolstoy I, Kropinski AM, Moraru C (2020) Create one new genus (Yonseivirus) including three new species (Caudovirales: Siphoviridae) https://ictv.global/ictv/proposals/ 2020.184B.R.Yonseivirus.zip.Phage.Canada@gmail.com

250. Wittmann J, Adriaenssens EM, Kropinski AM (2020) Create one new genus (Zicotriavirus) including two new species (Caudovirales: Schitoviridae) https://ictv.global/ictv/proposals/2020. 185B.R.Zicotriavirus.zip.Johannes.Wittmann@dsmz.de

251. Krupovic M, Kuhn JH, Wang F, Baquero DP, Egelman EH, Koonin EV, Prangishvili D (2020) Create one new realm (Adnaviria) for classification of filamentous archaeal viruses with linear dsDNA genomes https://ictv.global/ictv/proposals/2020. 186B.R.Adnaviria.zip.mart.krupovic@pasteur.fr

252. Bischoff V, Adriaenssens EM, Kropinski AM, Duhaime M, Moraru C (2020) Create one new family (Zobellviridae) including one new subfamily (Cobavirinae), seven new genera and 12 new species (Caudovirales) https://ictv.global/ictv/propo sals/2020.187B.R.Zobellviridae.zip. liliana.cristina.moraru@ uni-oldenburg.de

253. Adriaenssens EM, Tolstoy I, Kropinski AM, Moraru C, Wittmann J (2020) Create one new genus (Zurivirus) including one new species (Caudovirales: Schitoviridae) https://ictv.global/ictv/ proposals/2020.188B.R.Zurivirus.zip. jow12@dsmz.de

254. Adriaenssens EM (2021) Delete the Excel modules of proposals 2020.121B and 2020.126B and replace them with the 
2020.146B Excel module (Caudovirales: Schitoviridae) https:// ictv.global/ictv/proposals/2020.189B.R.Expedited_correction. docx. Evelien.adriaenssens@quadram.ac.uk

255. Adriaenssens EM (2021) Correct typographical errors in proposal 2020.005B Excel module (Caudovirales: Ackermannviridae) https://ictv.global/ictv/proposals/2020.190B.R.Corre ctions_Ackermannviridae.zip.Evelien.adriaenssens@ quadram. ac.uk

256. Ayllón MA, Turina M, Xie J, Jiang D, Marzano S-Y, Nerva L, Donaire L (2020) Create two new genera (Rhizoulivirus and Penoulivirus) and 25 new species (Ourlivirales: Botourmiaviridae) https://ictv.global/ictv/proposals/2020.001F.R.Botourmiav iridae.zip.mariaangeles.ayllon@upm.es

257. Vainio EJ, Chiba S, Nibert ML, Roossinck MJ, Sabanadzovic S, Suzuki N, Xie J (2020) Create one new family Curvulaviridae, one new genus Orthocurvulavirus and eight new species https:// ictv.global/ictv/proposals/2020.002F.R.Curvulaviridae.zip. eeva. vainio@luke.fi

258. Kotta-Loizou I, Castón JR, Coutts RHA, Hillman BI, Jiang D, Kim D-H, Moriyama H, Suzuki N (2020) Create six new species in the genus Alphachrysovirus (Ghabrivirales: Chrysoviridae) https://ictv.global/ictv/proposals/2020.003F.R.Chrysoviridae.zip. i.kotta-loizou13@imperial.ac.uk

259. Jiang D, Ayllón MA, Marzano S-Y (2020) Create seven new genera (Mononegavirales: Mymonaviridae) https://ictv.global/ ictv/proposals/2020.004F.R.Mymona.zip.daohongjiang@mail. hzau.edu.cn

260. Varsani A, Krupovic M (2020) Create one new genus, rename 73 species and create 164 new species (Geplafuvirales: Genomoviridae) https://ictv.global/ictv/proposals/2020.005F.R.Genomoviri dae.zip.Arvind.varsani@asu.edu

261. Gronenborn B, Varsani A, Randles JW, Vetten HJ, Thomas JE (2020) Create a new subfamily (Petromoalphasatellitinae) including four new genera and seven new species (Alphasatellitidae) https://ictv.global/ictv/proposals/2020.001P.R.Petromoalp hasatellitinae_nsf.zip. bgronenborn@gmail.com

262. Varsani A, Martin DP, Lett J-M, Roumagnac P (2020) Create nine new species and three new genera (Alphasatellitidae) https://ictv.global/ictv/proposals/2020.002P.R.Alphasatellitidae_ 3ng_9nsp.zip. Arvind.Varsani@asu.edu

263. Teycheney P-Y, Umber M, Geering ADW (2020) Create eight new species in the genus Badnavirus, one new species in the genus Cavemovirus, and one new genus (Ortervirales: Caulimoviridae) https://ictv.global/ictv/proposals/2020.003P.R.Cauli moviridae_1ng_9nsp.zip.teycheney@cirad.fr

264. Fuchs M, Bar-Joseph M, Candresse T, Maree HJ, Melzer MJ, Menzel W, Minafra A, Sabanadzovic S (2020) Create three new species in the genera Closterovirus, Ampleovirus and Velarivirus (Martellivirales: Closteroviridae) https://ictv.global/ictv/propo sals/2020.004P.R.Closteroviridae_3nsp.zip.mf13@cornell.edu

265. Lett J-M, Martin DP, Roumagnac P, Varsani A (2020) Create four new species in the genus Mastrevirus (Geminiviridae) https:// ictv.global/ictv/proposals/2020.005P.R.Mastrevirus_4nsp.zip. jean-michel.lett@cirad.fr

266. Fiallo-Olivé E, Navas-Castillo J (2020) Create 22 species and abolish one species in the genus Begomovirus (Geminiviridae) https://ictv.global/ictv/proposals/2020.006P.R.Begomovirus_ 22nsp.zip.efiallo@eelm.csic.es

267. Fiallo-Olivé E, Navas-Castillo J (2020) Create one species in the genus Deltasatellite (Tolecusatellitidae) https://ictv.global/ictv/ proposals/2020.007P.R.Deltasatellite_1nsp.zip. efiallo@eelm. csic.es

268. Roumagnac P, Varsani A, Martin DP, Lett J-M (2020) Create five new genera and 11 new species (Geplafuvirales: Geminiviridae) https://ictv.global/ictv/proposals/2020.008P.R.Geminiviridae_ 5ng_11nsp.zip.philippe.roumagnac@cirad.fr
269. Fiallo-Olivé E, Navas-Castillo J (2020) Create 61 species and abolish three species in the genus Betasatellite (Tolecusatellitidae) https://ictv.global/ictv/proposals/2020.009P.R.Betasatellite_ 61nsp.zip.efiallo@eelm.csic.es

270. Melzer M, Freitas-Astua J, Gang W, Rodrigues JCV, Roy A (2020) Create one new species in the genus Blunervirus (Martellivirales: Kitaviridae) https://ictv.global/ictv/proposals/2020. 010P.R.Kitaviridae_1nsp.zip.melzer@hawaii.edu

271. Ramos-González PL, Chabi-Jesus C, Freitas-Astúa J (2020) Create one new species in the genus Cilevirus (Martellivirales: Kitaviridae) https://ictv.global/ictv/proposals/2020.011P.R.Kitav iridae_1nsp.zip.juliana.astua@embrapa.br

272. Wang Y, Zhai L, Wen S, Yang Z, Wang G, Hong N (2020) Create one new species (Actinidia virus 2) in the genus Emaravirus (Bunyavirales: Fimoviridae) https://ictv.global/ictv/proposals/ 2020.012P.R.Emaravirus_AcV-2.zip.whni@mail.hzau.edu.cn

273. von Bargen S, Al Kubrusli R, Gaskin T, Fürl S, Hüttner F, Blystad D, Karlin DG, Jalkanen R, Büttner C (2020) Create one new species (Aspen mosaic-associated virus) in the genus Emaravirus (Bunyavirales: Fimoviridae) https://ictv.global/ictv/propo sals/2020.013P.R.Emaravirus_AsMaV.zip.susanne.von.bargen@ agrar.hu-berlin.de

274. Wang YQ, Yu S, Cao MJ, Cheng Q, Wu JX, Hu T (2020) Create one new species (Lilac chlorotic ringspot-associated virus) in the genus Emaravirus (Bunyavirales: Fimoviridae) https://ictv. global/ictv/proposals/2020.014P.R.Emaravirus_LiCRaV.zip. taohu1989@zju.edu.cn

275. Ilyas M, Avelar S, Schuch K, Brown JK (2020) Create one new species (Palo verde broom virus) in the genus Emaravirus (Bunyavirales: Fimoviridae) https://ictv.global/ictv/proposals/2020. 015P.R.Emaravirus_PVBV.zip. jbrown@ag.arizona.edu

276. Liu H, Wang G, Yang Z, Wang Y, Zhang Z, Li L, Waqas M, Zhang J, Xu L, Qi L, Hong J, Hong N (2020) Create one new species (Pear chlorotic leaf spot-associated virus) in the genus Emaravirus (Bunyavirales: Fimoviridae) https://ictv.global/ictv/ proposals/2020.016P.R.Emaravirus_PCLSaV.zip.whni@mail. hzau.edu.cn

277. Olmedo-Velarde A, Park AC, Sugano J, Uchida JY,Kawate M, Borth WB, Hu JS, Melzer MJ (2020) Create one new species (Ti ringspot-associated virus) in the genus Emaravirus (Bunyavirales: Fimoviridae) https://ictv.global/ictv/proposals/2020. 017P.R.Emaravirus_TiRSaV.zip.melzer@hawaii.edu

278. Peracchio C, Forgia M, Chiapello M, Vallino M, Turina M, Ciuffo M (2020) Create one new species (Camellia japonicaassociated virus 1) in the genus Emaravirus (Bunyavirales: Fimoviridae) https://ictv.global/ictv/proposals/2020.018P.R. Emaravirus_CjaV-1.zip. carlota.peracchio@ipsp.cnr.it

279. Peracchio C, Forgia M, Chiapello M, Vallino M, Turina M, Ciuffo M (2020) Create one new species (Camellia japonicaassociated virus 2) in the genus Emaravirus (Bunyavirales: Fimoviridae) https://ictv.global/ictv/proposals/2020.019P.R. Emaravirus_CjaV-2.zip.carlota.peracchio@ipsp.cnr.it

280. Yang C, Zhang S, Han T, Fu J, Di Serio F, Cao M (2020) Create one new species (Jujube yellow mottle-associated virus) in the genus Emaravirus (Bunyavirales: Fimoviridae) https://ictv. global/ictv/proposals/2020.020P.R.Emaravirus_JYMaV.zip. caomengji@cric.cn

281. Kubota K, Usugi T, Tomitaka Y, Shimomoto Y, Shigeharu T, Kadono F, Yanagisawa H, Chiaki Y, Tsuda S (2020) Create one new species (Perilla mosaic virus) in the genus Emaravirus (Bunyavirales: Fimoviridae) https://ictv.global/ictv/proposals/ 2020.021P.R.Emaravirus_PerMV.zip.kubotak@affrc.go.jp

282. Gronenborn B, Randles JW, Vetten HJ, Thomas JE (2020) Create one new family (Metaxyviridae) with one new genus (Cofodevirus) and one species (Coconut foliar decay virus) moved from the family Nanoviridae (Mulpavirales) https://ictv.global/ictv/ 
proposals/2020.022P.R.Metaxyviridae_nf.zip. bgronenborn@ gmail.com

283. Thomas JE, Gronenborn B, Vetten H-J, Heydarnejad J, Varsani A, Roumagnac P (2020) Create four new species in the genus Nanovirus (Mulpavirales: Nanoviridae) https://ictv.global/ictv/ proposals/2020.023P.R.Nanoviridae_4nsp.zip. j.thomas2@ uq.edu.au

284. Wylie SJ, Inoue-Nagata AK, Jordan R, Kreuze JF, Li F, LopezMoya JJ, Makinen K, Ohshima K (2020) Create seven new species in the genus Potyvirus (Patatavirales: Potyviridae) https:// ictv.global/ictv/proposals/2020.024P.R.Potyviridae_7nsp.zip. s.wylie@murdoch.edu.au

285. Sõmera M, Hébrard E (2020) Create one new species in the genus Sobemovirus (Sobelivirales: Solemoviridae) https://ictv.global/ ictv/proposals/2020.025P.R.Solemoviridae_1nsp.zip. merike. somera@taltech.ee

286. Scheets K, Miller WA, Sõmera, M (2020) Abolish the family Luteoviridae (Tolivirales) and move its genera to the families Tombusviridae (Tolivirales) and Solemoviridae (Sobelivirales) https://ictv.global/ictv/proposals/2020.026P.R.Abolish_Luteo viridae.zip.kay.scheets@okstate.edu

287. Qiu YJ, Zhang S, Zerbini FM, Cao MJ (2020) Create one new species (Paper mulberry leaf curl virus 1) in the genus Mulcrilevirus and one new species (Paper mulberry leaf curl virus 2) in the genus Citlodavirus (Geplafuvirales: Geminiviridae) https:// ictv.global/ictv/proposals/2020.027P.R.Geminiviridae_2nsp.zip. caomengji@cric.cn

288. Nemchinov LG (2020) Create one new species in the genus Marafivirus (Tymovirales: Tymoviridae) https://ictv.global/ictv/ proposals/2020.028P.R.Tymoviridae_1nsp.zip.lev.nemchinov@ usda.gov

289. Kreuze J, Candresse T, Hammond J, Menzel W, Ryu KH, Vaira AM, Zavriev S (2020) Create eight new species in the genus Potexvirus and one new species in the genus Allexivirus (Tymovirales: Alphaflexiviridae) https://ictv.global/ictv/proposals/2020. 029P.R.Alphaflexiviridae_9nsp.zip.j.kreuze@cgiar.org

290. Hammond J, Adams I, Fox A, Blouin AG (2020) Abolish one species in the genus Potexvirus (Tymovirales: Alphaflexiviridae) https://ictv.global/ictv/proposals/2020.030P.R.Alphaflexiviridae_ ab1sp.zip. john.hammond@usda.gov

291. Kreuze J, Candresse T, Hammond J, Menzel W, Ryu KH, Vaira AM, Zavriev S (2020) Abolish the genus Mandarivirus, create one new subgenus (Mandarivirus) in the genus Potexvirus and one new subgenus (Acarillexivirus) in the genus Allexivirus (Tymovirales: Alphaflexiviridae) https://ictv.global/ictv/propo sals/2020.031P.R.Alphaflexiviridae_ab1gen_2nsg.zip.j.kreuze@ cgiar.org

292. Wu JX, Zhang S, Zhou CY, Cao MJ (2020) Create one new species in the subgenus Mandarivirus, genus Potexvirus (Tymovirales: Alphaflexiviridae) https://ictv.global/ictv/proposals/2020. 032P.R.Mandarivirus_1nsp.zip.caomengji@cric.cn

293. Scheets K, Jordan R (2020) Create two new species in the genus Pelarspovirus (Tolivirales: Tombusviridae) https://ictv. global/ictv/proposals/2020.033P.R.Pelarspovirus_2nsp.zip. kay. scheets@okstate.edu

294. Scheets K (2020) Create two new species in genus Umbravirus (Tolivirales: Tombusviridae) https://ictv.global/ictv/proposals/ 2020.034P.R.Umbravirus_2nsp.zip.kay.scheets@okstate.edu

295. Scheets K (2020) Create one new species in the genus Alphacarmovirus (Tolivirales: Tombusviridae) https://ictv.global/ ictv/proposals/2020.035P.R.Alphacarmovirus_1nsp.zip. kay. scheets@okstate.edu

296. Scheets K (2020) Create one new species in the genus Aureusvirus (Tolivirales: Tombusviridae) https://ictv.global/ictv/propo sals/2020.036P.R.Aureusvirus_1nsp.zip. kay.scheets@okstate. edu
297. Suzuki N, Rubino L (2021) Correct the name of Sitke waterborne virus to Sikte waterborne virus (Tolivirales: Tombusviridae) https://ictv.global/ictv/proposals/2020.037P.R.Correction_ Tombusviridae.zip. luisa.rubino@ipsp.cnr.it

298. Andrewes CH (1951) Viruses and Linnaeus. Acta Path Micr Scand 28:211-225

299. Gibbs AJ, Harrison BD, Watson DH, Wildy P (1966) What's in a virus name? Nature 209:450-454

300. Lwoff A (1967) Principles of classification and nomenclature of viruses. Nature 215:13-14. https://doi.org/10.1038/215013a0

301. van Regenmortel MH (2000) On the relative merits of italics, Latin and binomial nomenclature in virus taxonomy. Arch Virol 145:433-441. https://doi.org/10.1007/s007050050036

302. Dijkstra J, Khan JA (2002) The current naming of plant viruses: a critical appraisal. Arch Virol 147:2251-2253. https://doi.org/ 10.1007/s007050200051

303. van Regenmortel MH, Mahy BW (2004) Emerging issues in virus taxonomy. Emerg Infect Dis 10:8-13. https://doi.org/10.3201/ eid1001.030279

304. Postler TS, Clawson AN, Amarasinghe GK, Basler CF, Bavari S, Benko M, Blasdell KR, Briese T, Buchmeier MJ, Bukreyev A, Calisher CH, Chandran K, Charrel R, Clegg CS, Collins PL, Juan Carlos T, Derisi JL, Dietzgen RG, Dolnik O, Dürrwald R, Dye JM, Easton AJ, Emonet S, Formenty P, Fouchier RAM, Ghedin E, Gonzalez JP, Harrach B, Hewson R, Horie M, Jiang D, Kobinger G, Kondo H, Kropinski AM, Krupovic M, Kurath G, Lamb RA, Leroy EM, Lukashevich IS, Maisner A, Mushegian AR, Netesov SV, Nowotny N, Patterson JL, Payne SL, PaWeska JT, Peters CJ, Radoshitzky SR, Rima BK, Romanowski V, Rubbenstroth D, Sabanadzovic S, Sanfaçon H, Salvato MS, Schwemmle M, Smither SJ, Stenglein MD, Stone DM, Takada A, Tesh RB, Tomonaga K, Tordo N, Towner JS, Vasilakis N, Volchkov VE, Wahl-Jensen V, Walker PJ, Wang LF, Varsani A, Whitfield AE, Zerbini FM, Kuhn JH (2017) Possibility and challenges of conversion of current virus species names to Linnaean binomials. Syst Biol 66:463-473. https://doi.org/10.1093/sysbio/syw096

305. Siddell SG, Walker PJ, Lefkowitz EJ, Mushegian AR, Dutilh BE, Harrach B, Harrison RL, Junglen S, Knowles NJ, Kropinski AM, Krupovic M, Kuhn JH, Nibert ML, Rubino L, Sabanadzovic S, Simmonds P, Varsani A, Zerbini FM, Davison AJ (2020) Binomial nomenclature for virus species: a consultation. Arch Virol 165:519-525. https://doi.org/10.1007/s00705-019-04477-6.Errat um.In:ArchVirol.2020165:1263-1264

306. Siddell SG, Walker PJ, Lefkowitz EJ, Mushegian AR, Dutilh BE, Harrach B, Harrison RL, Junglen S, Knowles NJ, Kropinski AM, Krupovic M, Kuhn JH, Nibert ML, Rubino L, Sabanadzovic S, Simmonds P, Varsani A, Zerbini FM, Davison AJ (2020) Correction to: Binomial nomenclature for virus species: a consultation. Arch Virol 165:1263-1264. https://doi.org/10.1007/s00705-02004555-0.Erratumfor:ArchVirol.2020Feb;165(2):519-525

307. https://talk.ictvonline.org/ictv1/f/taxonomic-opinions/3952/ binomial-species-names

308. Van Regenmortel MHV (2020) A brief history of the species concept in virology and an opinion on the proposal to introduce Linnaean binomial virus species names. Arch Virol 165:3073-3077. https://doi.org/10.1007/s00705-020-04795-0

309. Gibbs A (2020) Binomial nomenclature for virus species: a long view. Arch Virol 165:3079-3083. https://doi.org/10.1007/ s00705-020-04828-8

Publisher's Note Springer Nature remains neutral with regard to jurisdictional claims in published maps and institutional affiliations. 


\section{Authors and Affiliations}

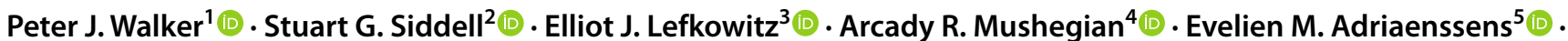

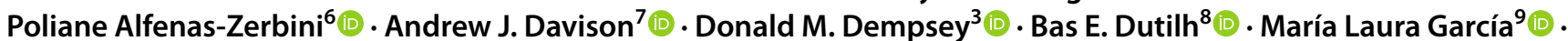

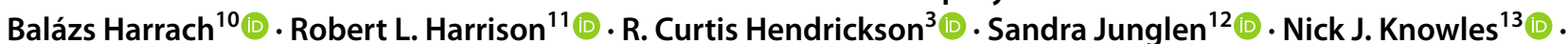

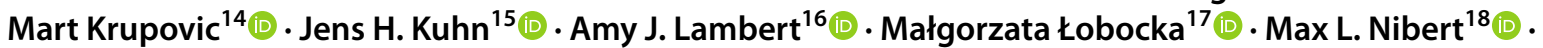

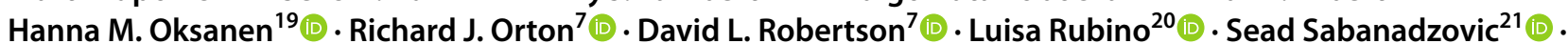

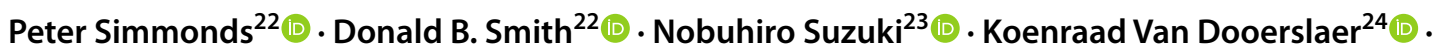
Anne-Mieke Vandamme ${ }^{25,26}$. Arvind Varsani ${ }^{27}\left[\right.$. Francisco Murilo Zerbini ${ }^{28}$

Peter J. Walker

Peter.Walker@uq.edu.au

Stuart G. Siddell

stuart.siddell@bristol.ac.uk

Elliot J. Lefkowitz

elliotl@uab.edu

Evelien M. Adriaenssens

evelien.adriaenssens@quadram.ac.uk

Poliane Alfenas-Zerbini

palfenas@ufv.br

Andrew J. Davison

andrew.davison@glasgow.ac.uk

Donald M. Dempsey

ddempsey@uab.edu

Bas E. Dutilh

bedutilh@gmail.com

María Laura García

garcia_m@biol.unlp.edu.ar

Balázs Harrach

harrach.balazs@vmri.hu

Robert L. Harrison

robert.1.harrison@ars.usda.gov

R. Curtis Hendrickson

curtish@uab.edu

Sandra Junglen

sandra.junglen@charite.de

Nick J. Knowles

nick.knowles@pirbright.ac.uk

Mart Krupovic

mart.krupovic@pasteur.fr

Jens H. Kuhn

kuhnjens@mail.nih.gov

Amy J. Lambert

ahk7@cdc.gov

Małgorzata Łobocka

lobocka@ibb.waw.pl

Max L. Nibert

mnibert@hms.harvard.edu

Hanna M. Oksanen

hanna.oksanen@helsinki.fi

Richard J. Orton

richard.orton@glasgow.ac.uk
David L. Robertson

david.1.robertson@glasgow.ac.uk

Luisa Rubino

luisa.rubino@cnr.it

Sead Sabanadzovic

ssabanadzovic@entomology.msstate.edu

Peter Simmonds

peter.simmonds@ndm.ox.ac.uk

Donald B. Smith

donald.smith.ictv@gmail.com

Nobuhiro Suzuki

nsuzuki@okayama-u.ac.jp

Koenraad Van Dooerslaer

vandoorslaer@arizona.edu

Anne-Mieke Vandamme

annemie.vandamme@kuleuven.be

Arvind Varsani

arvind.varsani@asu.edu

Francisco Murilo Zerbini

zerbini@ufv.br

1 School of Chemistry and Molecular Biosciences, The University of Queensland, St Lucia, QLD 4072, Australia

2 School of Cellular and Molecular Medicine, Faculty of Life Sciences, University of Bristol, University Walk, Bristol BS8 1TD, UK

3 Department of Microbiology, University of Alabama at Birmingham (UAB), BBRB 276, 845 19th St South, Birmingham, AL 35294-2170, USA

4 Division of Molecular and Cellular Biosciences, National Science Foundation, 2415 Eisenhower Avenue, Alexandria, VA 22314, USA

5 Quadram Institute Bioscience, Norwich Research Park, Norwich NR4 7UQ, UK

6 Departamento de Microbiologia, Universidade Federal de Viçosa, Viçosa, MG 36570-000, Brazil

7 MRC-University of Glasgow Centre for Virus Research, Sir Michael Stoker Building, 464 Bearsden Road, Glasgow G61 1QH, UK

8 Theoretical Biology and Bioinformatics, Department of Biology, Utrecht University, Padualaan 8, Room Z-509, $3584 \mathrm{CH}$ Utrecht, The Netherlands 
9 Instituto de Biotecnología y Biología Molecular, CCT-La Plata, CONICET, UNLP, calles 47 y 115 (1900), La Plata, Buenos Aires, Argentina

10 Veterinary Medical Research Institute, Hungária krt. 21, H-1143 Budapest, Hungary

11 Invasive Insect Biocontrol and Behavior Laboratory, USDA-ARS, 10300 Baltimore Avenue, Bldg 007 Barc-West, Beltsville, MD 20705, USA

12 Institute of Virology, Charité-Universitätsmedizin, corporate member of Free University Berlin, Humboldt-University Berlin, and Berlin Institute of Health, Germany, Berlin, Germany

13 The Pirbright Institute, Ash Road, Pirbright GU24 0NF, Surrey, UK

14 Department of Microbiology, Institut Pasteur, 25 rue du Dr Roux, 75015 Paris, France

15 NIH/NIAID/DCR/Integrated Research Facility at Fort Detrick (IRF-Frederick), B-8200 Research Plaza, Fort Detrick, Frederick, MD 21702, USA

16 Division of Vector-Borne Diseases, National Center for Emerging and Zoonotic Infectious Diseases, Centers for Disease Control and Prevention, Fort Collins, CO 80521, USA

17 Institute of Biochemistry and Biophysics of the Polish Academy of Sciences, 02-106, Warsaw, Poland

18 Department of Microbiology, Harvard Medical School, 77 Ave Louis Pasteur, Boston, MA 02115, USA

19 Molecular and Integrative Biosciences Research Programme, Faculty of Biological and Environmental Sciences, University of Helsinki, Viikinkaari 9, FI-00014 Helsinki, Finland
20 Istituto per la Protezione Sostenibile delle Piante, CNR, UOS Bari, Via Amendola 165/A, 70126 Bari, Italy

21 Department of Biochemistry, Molecular Biology, Entomology and Plant Pathology, Mississippi State University, 100 Old Hwy 12 Mail Stop 9775, Mississippi State, MS 39762, USA

22 Nuffield Department of Medicine, University of Oxford, Peter Medawar Building, South Parks Road, Oxford OX1 3SY, UK

23 Institute of Plant Science and Resources, Okayama University, Kurashiki, Okayama 710-0046, Japan

24 School of Animal and Comparative Biomedical Sciences, Department of Immunobiology, BIO5 Institute, and University of Arizona Cancer Center, Tucson, AZ 85721, USA

25 KU Leuven, Department of Microbiology, Immunology and Transplantation, Rega Institute for Medical Research, Clinical and Epidemiological Virology, Institute for the Future, 3000 Leuven, Belgium

26 Center for Global Health and Tropical Medicine, Unidade de Microbiologia, Instituto de Higiene e Medicina Tropical, Universidade Nova de Lisboa, Rua da Junqueira, 100, 1349-008 Lisboa, Portugal

27 The Biodesign Center for Fundamental and Applied Microbiomics, School of Life Sciences, Center for Evolution and Medicine, Arizona State University, P.O. Box 874701, Tempe, AZ 85287-4701, USA

28 Departamento de Fitopatologia/BIOAGRO, Universidade Federal de Viçosa, Viçosa, MG 36570-900, Brazil 